Noname manuscript No.

(will be inserted by the editor)

\title{
A non-local cross-diffusion model of population dynamics I: Emergent spatial and spatiotemporal patterns
}

\author{
Nick P. Taylor • Hyunyeon Kim • Andrew L. Krause • \\ Robert A. Van Gorder
}

Received: date / Accepted: date

\begin{abstract}
We extend a spatially non-local cross-diffusion model of aggregation between multiple species with directed motion toward resource gradients to include many species and more general kinds of dispersal. We first consider diffusive instabilities, determining that for directed motion along fecundity gradients, the model permits the Turing instability leading to colony formation and persistence provided there are three or more interacting species. We also prove that such patterning is not possible in the model under the Turing mechanism for two species under directed motion along fecundity gradients, confirming earlier findings in the literature. However, when the directed motion is not along fecundity gradients, for instance if foraging or migration is sub-optimal relative to fecundity gradients, we find that very different colony structures can emerge. This generalization also permits colony formation for two interacting species. In the advection - dominated case, aggregation patterns are more broad and global in nature, due to the inherent non-local nature of the advection which permits directed motion over greater distances, whereas in the diffusion - dominated case, more highly localized pattens and colonies develop, owing to the localized nature of random diffusion. We also consider the interplay between Turing patterning and spatial heterogeneity in resources. We find that for small spatial variations, there will be a combination of Turing patterns and patterning due to spatial forcing from the resources, whereas for large resource variations, spatial or spatiotemporal patterning can be modified greatly from what is predicted on homogeneous domains. For each of these emergent behaviors, we outline the theoretical mechanism leading to colony formation, and then provide numerical simulations to illustrate the results. We also discuss implications this model has for studies of directed motion in different ecological settings.
\end{abstract}

Keywords aggregation · directed motion · Turing instability · colony formation

\section{Introduction}

Since Turing's famous work on morphogenesis (Turing, 1952), the emergence of structure in reactiondiffusion models in and beyond developmental biology has been well studied (Kishimoto, 1982; Kishimoto et al, 1983; Kishimoto and Weinberger, 1985; Riaz et al, 2005) and in particular can provide a useful modelling framework for spatial ecology. Reaction-diffusion models can also exhibit complex spatiotemporal structures of more complexity than stationary patterned states, and these have been implicated as important hallmarks of ecological complexity (Malchow et al, 2007). However, in certain ecological contexts, reaction-diffusion models may not adequately describe how organisms move and disperse (Rowell, 2009). In particular, Rowell (2009) explains how models where species simply diffuse at random fail to explain certain ecological phenomena, and do not accurately reflect the non-Brownian motion of individuals. Rational or pseudo-rational movement along resource gradients is thought to reduce rates of expansion and lead to clustering and formation of colonies, distinct from what might be predicted in models where

N. P. Taylor

Department of Plant Sciences, University of Cambridge, Downing Street, Cambridge CB2 3EA United Kingdom H. Kim and A. L. Krause

Mathematical Institute, University of Oxford, Andrew Wiles Building, Radcliffe Observatory Quarter, Woodstock Road, Oxford OX2 6GG United Kingdom

R. A. Van Gorder

Department of Mathematics and Statistics, University of Otago, P.O. Box 56, Dunedin 9054, New Zealand

E-mail: rvangorder@maths.otago.ac.nz 
the only motion is through random diffusion. The concept of fitness dependent dispersal was discussed further by Armsworth and Roughgarden (2008), where models of clines with fitness-dependent dispersal were considered. This built on theoretical work of Grindrod (1988), as well as more recent work in the area of fitness dependent dispersal (Abrams, 2007; Abrams et al, 2007; Amarasekare, 2007; Armsworth and Roughgarden, 2005a,b; Hadany et al, 2004; Ruxton and Rohani, 1999). It has been suggested that time spent moving along resource gradients (more generally, searching out resources) can limit mobility of species, relative to if the species were to simply disperse randomly (Rowell, 2009). Rowell (2009) suggests that this dispersion along resource gradients may result in an increased likelihood of colony formation, and there is experimental evidence that advection in general can prevent extinction for some species (Hastings et al, 2011).

Shigesada et al (1979) introduced cross-diffusion models in order to model dispersal with movement up resource gradients or to avoid crowding. Indeed, the emergence of spatial patterning implying colony formation has been observed in a variety of cross diffusion models involving two populations (Lou and Ni, 1996a; Gambino et al, 2013; Ferreira et al, 2019; Mukherjee et al, 2019) as well as three or more (Tian et al, 2011; Lv et al, 2013; Kondratyev et al, 2016; Wen and Fu, 2016; Ma et al, 2017). Cantrell et al (2013) discuss random dispersal versus fitness-dependent dispersal, with explicit fitness dependence included in the dispersal terms (often, it is also prescribed as a modeling assumption). This is in contrast to Grindrod $(1988,1991)$, for which the optimal direction in which the populations advect is solved for using additional equations which depend on the fecundity gradient. In this way, one can view the model of Grindrod $(1988,1991)$ as spatially non-local, as one must solve for the advection direction in terms of the fecundity, which depends on the unknown population densities.

There are numerous facets of spatial population ecology for which mechanistic models of pattern formation can help elucidate fundamental processes of spatial organization. These include aggregation and coexistence of species (Murrell and Law, 2003), territoriality (Lewis and Murray, 1993; Potts and Lewis, 2014), and home range analysis (Moorcroft et al, 1999; Mitchell and Powell, 2004). Understanding directed motion is of particular importance in cases such as predators seeking prey ("prey-taxis") (Kareiva and Odell, 1987; Lee et al, 2009), and the opposite phenomenon of prey avoiding predators, and more generally competing species avoiding one another (sometimes called the "landscape of fear") (Laundré et al, 2010; Madin et al, 2011). In recent decades, so-called "movement ecology" has emerged to integrate information about individual organism movement with macroecological dispersal patterns, in order to conceptualize the role of movement across diverse ecosystem scales (Holden, 2006; Nathan, 2008). Contemporary empirical studies collect and analyze data regarding organism movement on scales from individual-level detail to hemispheric distributions (Allen and Singh, 2016; Taylor et al, 2017). Alongside such studies and statistical approaches for drawing meaningful inferences from them, mechanistic theoretical models play an important role in elucidating fundamental relationships between species distributions and individual-level movement (Lewis et al, 2013; Shepard et al, 2013; Potts and Lewis, 2019).

Aside from population ecology, there are a variety of other applications for mechanistic models of directed motion. There is a population genetics context for reaction-diffusion-advection models (Armsworth and Roughgarden, 2008; Bolnick and Otto, 2013; Richardson et al, 2014). Armsworth and Roughgarden (2008) discuss a way of modelling clines with fitness-dependent dispersal. Orr (2009) discusses how populations can get stuck at local adaptive peaks that may not be the highest peak on the fitness landscape, and thus not attain maximal fitness, whilst Bolnick and Otto (2013) describe a concept called 'migration load'. Dispersal of individuals away from the environment of their immediate ancestors is generally expected to reduce fitness. However, it is found that migration load can be reduced or even reversed if we allow preferential movement of individuals towards regions of higher fitness; this is analogous to the inclusion of advection in the reaction-diffusion-advection model when working within the genotype-phenotype context. Richardson et al (2014) discuss local adaptation. Similar concepts could be used in a reaction-diffusion-advection model with pattern formation in different regions of a fitness landscape indicating differences between individuals of the same species existing in different environments. Reaction-diffusion-advection models were used by Kondo and Miura (2010) to study the early development of animal embryos.

Potts and Lewis (2019) recently explored the setting of spatial dispersal on timescales smaller than birth and death processes, and hence could study a variety of different kinds of directed movement and their impact on spatial organization in the absence of non-spatial population interactions (reactions). In addition to diffusive (random) population dispersal, they investigated taxis of a population in response to the current or recent presence of a different population, sensed via either direct observation, markings or scents, or memory of past interactions. For $N=2$ distinct populations, they classify all emergent 
patterns as stationary, but show for $N \geq 3$ that spatiotemporal oscillations exist. We also note that there is a range of pure transport (i.e. no local reaction term) models of aggregating species which are conceptually similar to these diffusion-taxis models (Padrón, 1998, 2004).

Motivated by these various applications for reaction-diffusion-advection models which allow for directed or intelligent motion, in the present paper we are concerned with the dispersal and clustering of populations of multiple species interacting via an extension of the model proposed by Grindrod (1988) controlling the dispersal of individuals in single and multispecies communities (Grindrod, 1988, 1991). The work of Grindrod (1988) built on simpler models by Gurtin and MacCamy (1977) and Bertsch et al (1985), by modelling behaviors where individuals can respond to threats of predation and starvation, and responses to the environment occur on a much shorter timescale than births and deaths of individuals. This motion was modelled separately from random diffusion through an advection term, with each species moving along a fecundity gradient. The dynamics of such models in one spatial domain were considered in Grindrod (1988), and in two spatial dimensions in Kurowski et al (2017). Clustering and other interesting dynamics were observed on the finite one-dimensional spatial domain for short time scales, though it was shown in Kurowski et al (2017) that long-term colony formation does not persist in convex domains (although it may in non-convex domains). The evolution of conditional dispersal strategies in the context of competition between two species that are ecologically identical except in their dispersal mechanisms was considered in Chen et al (2008), and the spatial dependence of such dynamics was then considered in Hambrock and Lou (2009), when a spatially heterogeneous domain was considered. The dynamics of the two-species form of the model on heterogeneous domains resulting from spatially varying resources or hazards was also studied by Kurowski et al (2017), and it was shown that such spatial heterogeneity can result in colony formation through a mechanism distinct from the Turing instability.

In terms of more mathematically rigorous work on the model proposed by Grindrod (1988), the local existence and uniqueness of solutions for a one-species version of the model is discussed by Nasreddine (2012), with global existence demonstrated in one spatial dimension for two particular choices of the fecundity function $E$. Nasreddine (2014) then demonstrated global existence for the same two choices of $E$ in two spatial dimensions, again for a one-species model. We are aware of no corresponding results for multiple species models of the form discussed in (Grindrod, 1988, 1991; Kurowski et al, 2017).

Here we extend the model studied in (Grindrod, 1988, 1991; Kurowski et al, 2017) in a few ways. First, we consider more than two species or populations. Kurowski et al (2017) showed that pattern formation through the Turing mechanism does not occur for the two-species variant of this model. Patterning was shown on non-convex domains, but that is through a different mechanism (Matano and Mimura, 1983). In the present paper, we formulate a generalized model for any number of species, as this will be helpful in the study of spatial partitioning due to niche formation, wherein organisms spontaneously cluster into regions preferable to themselves. Second, solutions were assumed to be irrotational in Kurowski et al (2017), and this was primarily for computational simplicity. In the present paper we relax this assumption, and hence are able to arrive at a somewhat more general stability result in the case of two species. Finally, we relax the assumption that the non-local advection is strictly directed along fecundity gradients. Indeed, in real-world examples with limited information, migrations are based more on expectation than on complete knowledge of an optimal trajectory. Allowing the true motion to deviate from the optimal motion suggested by fecundity gradients allows one to study sub-optimal migrations, or migrations driven by factors other than fecundity gradients.

We study emergent pattern-forming behavior due to these generalizations, finding the following broad trends. When the number of populations is three or more, there can be Turing-like instabilities and hence pattern formation in the standard Grindrod-like model. For two species, the model is not of the activatorinhibitor form, and hence does not permit patterning through this mechanism. For three or more species in the presence of small spatial heterogeneity in resources, we find combinations of Turing patterns and patterns emergent from the forced heterogeneity. In the case where the directed motion is not along the fecundity gradients, we show that colony formation via a Turing-type instability is possible for two species models, and can occur in larger parameter regimes for more species. This may be a result of colony formation being an effective strategy when migration paths are sub-optimal. In this first part, we confine our discussion to a presentation of the model and its pattern-formation properties, and study traveling waves of migration and invasion in (Krause and Van Gorder, 2020).

We remark that the Lotka-Volterra functional responses use have both advantages and disadvantages. Such responses allow us to simplify our discussion of inter- and intra-population competition and predation in terms of a simple interaction structure (the community matrix). It is also the simplest nontrivial choice of a functional response, and is still used heavily in contemporary models across ecology and related areas (Ackland and Gallagher, 2004; Strobl et al, 2019; Zhao et al, 2019). However, it does 
not allow for Allee effects (Petrovskii et al, 2002; Morozov et al, 2006), or other kinds of nonlinear phenomena associated with more realistic functional responses, such as predator interference (Alonso et al, 2002). Hence this study should be viewed as an idealization which allows us to explore the interplay of competition and predator-prey interactions alongside directed motion in the simplest case first, before further extending these models to more realistic interactions.

The remainder of this paper is organized as follows. In Section 2, we outline an extension of the model formulated in Grindrod (1988) to the case of multiple species of domains of arbitrary dimension (although throughout this paper we shall focus on the case of two-dimensional spatial domains for illustrative purposes). We also propose a non-dimensional form of the model, and discuss how this model fits into the context of cross diffusion models. There are a variety of behaviors previously unexplored in this model, and discussing pattern-forming solution behaviors will be the focus of the remainder of the paper. In Section 3 we study diffusion driven instabilities which result in pattern formation when species are advected along fecundity gradients, showing that such instabilities only occur under the model for three or more species, and hence colony formation and persistence is possible through the Turing mechanism. We also give a succinct explanation for why there is no long-term patterning in convex domains as seen previously in Kurowski et al (2017). In Section 4 we discuss advection driven instabilities which result from directed motion along a gradient different from a fecundity gradient. Such motion may arise when species are not perfectly able to detect fecundity gradients, or if other dispersal strategies are employed. Interestingly, this instability mechanism results in pattern formation even if there are only two species. Finally, motivated by Kurowski et al (2017), we consider model solutions on spatially heterogeneous domains due to spatial resources and hazards in Section 5, focusing on how this spatial forcing modifies Turing patterns emergent in Section 3. We conclude by summarising some of the more interesting findings in Section 6, and discussing general insights into ecological models involving directed motion.

\section{Competition model with advection toward resource gradients}

A dimensional form of Grindrod's two-species competition model (Grindrod, 1988, 1991) generalized to many species and to arbitrary spatial domains reads

$$
\begin{gathered}
\frac{\partial \hat{u}_{\ell}}{\partial \hat{t}}=\hat{\delta}_{\ell} \nabla^{2} \hat{u}_{1}-\hat{\mu}_{\ell} \nabla \cdot\left(\hat{u}_{\ell} \hat{\mathbf{w}}_{\ell}\right)+\hat{r} \hat{u}_{\ell} \hat{E}_{\ell}\left(\hat{u}_{1}, \ldots, \hat{u}_{n}\right), \\
-\hat{\epsilon}_{\ell} \nabla^{2} \hat{\mathbf{w}}_{\ell}+\hat{\mathbf{w}}_{\ell}=\nabla \hat{F}_{\ell}\left(\hat{u}_{1}, \ldots, \hat{u}_{n}\right),
\end{gathered}
$$

$\ell=1,2, \ldots, n$ where $n \geq 1$ denotes the number of species. Here $\hat{u}_{\ell}(\mathbf{x}, t)$ denotes the population density of species $\ell$ at $\mathbf{x} \in \Omega \subseteq \mathbb{R}^{m}(m=1,2,3), t>0, \hat{\mathbf{w}}_{\ell}$ is the average velocity of those individuals of population $\ell$ dispersing, and $\hat{E}_{\ell}$ is the projected net rate of reproduction (fecundity) with $\hat{\delta}_{\ell}, \hat{r}, \hat{\mu}_{\ell}, \hat{\epsilon}_{\ell} \geq 0$. The functions $\hat{F}_{\ell}$ denote gradient potentials which will direct the advective motion of each species. Note that $\hat{\epsilon}_{\ell}$ can be considered as the accuracy of determining the local fecundity gradient or the efficiency of using the information about the abundance of a resource, or the existence of a hazard. Then $-\hat{\epsilon}_{\ell} \nabla^{2} \hat{\mathbf{w}}_{\ell}$ can be seen as an effective local average which implies an imprecise knowledge of fitness gradients and helps regularize the advective dynamics.

In the special case of $\nabla \hat{F}_{\ell}=\nabla \hat{E}_{\ell}$, we recover the model studied in Grindrod $(1988,1991)$. If $\hat{\varepsilon}=0$, then the system reduces to a system of reaction-diffusion-advection PDEs. The results of Theorem 2.6 of Nasreddine (2012) imply convergence of solutions uniformly in $t$ for $\hat{\varepsilon} \rightarrow 0$ of the one species model in one dimension with spatially homogeneous logistic kinetics. Assuming that the well-posedness results from Nasreddine (2014) can be extended to the two-species case given by (1), along with some technicalities about the compactness of solutions, we expect this simplification to be asymptotically justified for $\hat{\varepsilon}_{\ell} \ll 1$, since $\hat{\varepsilon}_{\ell}=0$ implies $\hat{\mathbf{w}}_{\ell}=\nabla \hat{E}_{\ell}$.

In Grindrod $(1988,1991), \hat{E}_{\ell}$ were taken as

$$
\hat{E}_{\ell}\left(\hat{u}_{1}, \hat{u}_{2}, \ldots, \hat{u}_{n}\right)=\hat{A}_{\ell}-\sum_{j=1}^{n} \hat{a}_{\ell j} u_{j} .
$$

This choice results in (1a) taking the form of a spatially extended Lotka-Volterra model. The non-negative parameters $\hat{A}_{\ell}$ express how the populations exploit a spatially distributed resource. If $\hat{A}_{\ell}$ is large, the populations have a greater chance to survive. We take $\hat{a}_{\ell \ell}>0$ for all $\ell$ to signify that a species' growth will be eventually limited by intraspecific competition. For $\ell \neq j, \hat{a}_{\ell j}$ will be positive if the species $\ell$ is 
harmed by species $j$ (for example if species $\ell$ is preyed upon by species $j$ or in competition with species $j$ ), and negative if species $\ell$ benefits from species $j$ (for example if species $\ell$ is a predator preying upon species $j$, or mutualism if both $\hat{a}_{\ell j}$ and $\hat{a}_{j \ell}$ are negative). To simplify the presentation later, we will neglect cases of mutualism, though we remark that some multispecies models have implicated it as a route to pattern formation (Tian et al, 2011). We assume that if species $\ell$ is an intraguild predator, it is attracted to herbivorous prey species $j$, and in direct competition for resources with it (we model both competition and predation using the same term in the prey equation). This is because it can eat species $j$. If species $\ell$ and $j$ do not interact directly, then $\hat{a}_{\ell j}=\hat{a}_{j \ell}=0$. See Table 1 for examples of parameter signs depending on the ecological context.

\begin{tabular}{|c|c|c|c|c|}
\hline & Predator $\ell$, Prey $j$ & Intraguild Predator $\ell$, Prey $j$ & Competition & No interaction \\
\hline$\hat{a}_{\ell j}$ & - & - & + & 0 \\
\hline$\hat{a}_{j \ell}$ & + & + & + & 0 \\
\hline
\end{tabular}

Table 1 Sign of the interspecies interaction parameters for a variety of behaviors. A negative parameter denotes attraction, whereas a positive parameters denotes repulsion. Mathematically, a predator-prey relationship has the prey as the only source of food for the predator $\left(A_{\ell}=0\right)$, and an intraguild predator-prey relationship allows the predator to live on resources in the domain in addition to the prey $\left(A_{\ell}>0\right)$.

Similarly, one may take

$$
\hat{F}_{\ell}\left(\hat{u}_{1}, \hat{u}_{2}, \ldots, \hat{u}_{n}\right)=-\sum_{j=1}^{n} \hat{b}_{\ell j} \hat{u}_{j} .
$$

If $\hat{b}_{\ell j}=\hat{a}_{\ell j}$, then we recover the special case $\nabla \hat{F}_{\ell}=\nabla \hat{E}_{\ell}$ which has been studied previously for models with one or two species.

Regarding boundary and initial conditions, we use Neumann boundary conditions, so that there is no flux of any of the populations in or out of our domain $\Omega$. Let the normal vector to the boundary $\partial \Omega$ be denoted by $\mathbf{n}$. Then we have

$$
\mathbf{n} \cdot \hat{\mathbf{w}}_{\ell}=\mathbf{n} \cdot \nabla \hat{u}_{\ell}=0,
$$

for $\ell=1, \ldots, n$, and it is easy to see that this implies no-flux conditions for each species. The initial conditions are taken to be

$$
\hat{\mathbf{u}}(\mathbf{x}, 0)=\hat{\mathbf{u}}_{0}(\mathbf{x}) \geq 0
$$

\subsection{Dimensionless model}

In what follows, we non-dimensionalize the model in order to reduce the parameter space. Introducing the scalings $\hat{u}_{\ell}(\hat{\mathbf{x}}, \hat{t})=u_{\ell}(\mathbf{x}, t), \hat{t}=\frac{1}{\hat{r}} t, \hat{\mathbf{x}}=\hat{L} \mathbf{x}$ (where $\hat{L}>0$ is a characteristic length scale), $\hat{\mathbf{w}}_{\ell}=\frac{1}{\hat{L}} \mathbf{w}_{\ell}$ and defining the dimensionless parameters $\delta_{\ell}=\frac{\hat{\delta}_{\ell}}{\hat{r} \hat{L}^{2}}>0, \mu_{\ell}=\frac{\hat{\mu}_{\ell}}{\hat{r} \hat{L}^{2}} \geq 0, \epsilon_{\ell}=\frac{\hat{\epsilon}_{\ell}}{\hat{L}^{2}} \geq 0$, we obtain the dimensionless model

$$
\begin{gathered}
\frac{\partial u_{\ell}}{\partial t}=\delta_{\ell} \nabla^{2} u_{\ell}-\mu_{\ell} \nabla \cdot\left(u_{\ell} \mathbf{w}_{\ell}\right)+u_{\ell} E_{\ell}, \\
-\epsilon_{\ell} \nabla^{2} \mathbf{w}_{\ell}+\mathbf{w}_{\ell}=\nabla F_{\ell}, \\
E_{\ell}\left(u_{1}, u_{2}, \ldots, u_{n}\right)=A_{\ell}-\sum_{j=1}^{n} a_{\ell j} u_{j}, \\
F_{\ell}\left(u_{1}, u_{2}, \ldots, u_{n}\right)=-\sum_{j=1}^{n} b_{\ell j} u_{j},
\end{gathered}
$$

for $\ell=1, \ldots, n$. Dimensionless boundary and initial conditions are

$$
\begin{gathered}
\mathbf{n} \cdot \mathbf{w}_{\ell}=\mathbf{n} \cdot \nabla u_{\ell}=0, \\
\mathbf{u}(\mathbf{x}, 0)=\mathbf{u}_{0}(\mathbf{x}) \geq 0 .
\end{gathered}
$$

We will assume $a_{i i}>0$ for all $i$ so that each species undergoes intraspecific competition, although the off-diagonal components can take either sign, depending on the dynamics being modelled. One can scale the density of each species to reduce some of the parameters in the kinetic functions, but we will not do so for generality and simplicity. 


\subsection{Relation to cross-diffusion models}

Before studying the system (6) in depth, we shall first comment on relations between our model and cross diffusion models. Cross-diffusion models have seen a variety of applications in the literature (Burger et al, 2010; Lou and Ni, 1996b), and have been shown to be of specific ecological relevance (Chen and Peng, 2004; Dubey et al, 2001; Kuznetsov et al, 1994; Peng et al, 2008; Shigesada et al, 1979; Wang et al, 2011).

In the $\epsilon_{\ell}=0$ limit, we can write $\mathbf{w}_{\ell}=\nabla F_{\ell}\left(u_{1}, \ldots, u_{n}\right)$. Placing this back into the reaction-diffusion equations, we obtain

$$
\frac{\partial u_{\ell}}{\partial t}=\nabla \cdot\left(\delta_{\ell} \nabla u_{\ell}+\mu_{\ell} \sum_{j=1}^{n} b_{\ell j} u_{\ell} \nabla u_{j}\right)+u_{\ell}\left(A_{\ell}-\sum_{j=1}^{n} a_{\ell j} u_{j}\right)
$$

which is a local cross-diffusion system. Related models have been shown to admit diffusion driven instabilities (Gambino et al, 2008, 2013; Lou et al, 2015; Mukherjee et al, 2019).

Given that $\epsilon_{\ell}$ is sufficiently small, note that $(6 \mathrm{~b})$ can be solved if one obtains the Green's function for $-\varepsilon_{\ell} \nabla^{2} \mathbf{w}_{\ell}+\mathbf{w}_{\ell}$, resulting in a non-local formulation. Alternatively, one may consider a formal expansion in small $\varepsilon_{\ell}$, of the form

$$
\mathbf{w}_{\ell}=\mathbf{w}_{\ell, 0}+\varepsilon_{\ell} \mathbf{w}_{\ell, 1}+\varepsilon_{\ell}^{2} \mathbf{w}_{\ell, 2}+\cdots
$$

At lowest order, note that from this expansion we recover the $\varepsilon_{\ell}=0$ limit, $\mathbf{w}_{\ell, 0}=\nabla F_{\ell}$. Placing the expansion (8) back into (6b), and matching powers of $\varepsilon_{\ell}$, we arrive at the formal expansion

$$
\mathbf{w}_{\ell}=\sum_{q=0}^{\infty} \varepsilon_{\ell}^{q} \nabla^{2 q} \nabla F_{\ell}\left(u_{1}, \ldots, u_{n}\right)=\nabla \sum_{q=0}^{\infty} \varepsilon_{\ell}^{q} \nabla^{2 q} F_{\ell}\left(u_{1}, \ldots, u_{n}\right),
$$

which we suppose will converge for small enough $\varepsilon_{\ell}$. Placing (9) back into the reaction-diffusion equations (6a), we obtain

$$
\frac{\partial u_{\ell}}{\partial t}=\nabla \cdot\left(\delta_{\ell} \nabla u_{\ell}+\mu_{\ell} \sum_{q=0}^{\infty} \varepsilon_{\ell}^{q} u_{\ell} \nabla^{2 q+1} F_{\ell}\left(u_{1}, \ldots, u_{n}\right)\right)+u_{\ell} E_{\ell}\left(u_{1}, \ldots, u_{n}\right)
$$

which is a non-local cross-diffusion system. We remark that non-local cross-diffusion models have been of increasing interest in the literature for a variety of biological and ecological applications (Fontbona and Méléard, 2015; Galiano, 2011; Hillen and Painter, 2009). Many models using dispersal kernels to capture non-random movement do not admit specific mechanistic interpretations for the individuals' movement within a population (Nathan and Muller-Landau, 2008; Lutscher, 2019). One benefit of our approach is that for (6) the non-locality associated to this cross-diffusion mechanism is encoded into the $\mathbf{w}_{\ell}$ functions. Although the dimension of the state space is higher, one can consider (6) as a purely local model encoding these non-local dispersal mechanisms. We also view (6) as easier to interpret as each term has a clear ecological significance.

\subsection{Summary and assumptions}

In what follows, we shall study the behavior of solutions emergent from (6), extending some of what was seen in the aforementioned literature, and showing new behaviors not previously studied or observed in such models. We will also suggest some plausible ecological interpretations, carefully noting the limitations inherent in our choice of functional response and other modelling assumptions. While the original model of Grindrod (1988) was designed for competition between two species utilizing similar resources, the reaction kinetics can be easily modified to account for predator-prey or intraguild predator - prey dynamics (Kurowski et al, 2017).

In Section 3, we obtain conditions for which spatial motion drives the spatially uniform steady state unstable though the Turing mechanism. We focus on the case of directed advection toward fecundity gradients, hence taking $\nabla F_{\ell}=\nabla E_{\ell}$ for all $\ell=1, \ldots, n$. We obtain the analytic criteria for a Turing instability, and then use this to motivate parameter regimes for which we carry out numerical simulations. We show that such instabilities only occur under the model for three or more species. We also prove that such patterning is not possible under the Turing mechanism for two species, generalizing a finding 
of Kurowski et al (2017). We focus much of our work in Section 3 on the case of three species, as this provides the simplest model which is sufficient to produce generic patterning behaviors.

Although advection toward resource gradients as calculated from fecundity are seemingly optimal in many scenarios, in reality this involves each species having complete information, which is somewhat unrealistic. Rather, it is more likely that each species would approximate this optimal fecundity gradient, with varying degrees of success. Furthermore, there may be other spatial cues or mitigating factors dictating behavior and hence motion of each species, with motion directed along another gradient altogether (e.g. memory or presence cues, as described in Potts and Lewis (2019)). For these reasons, we explore the model (6) for the case $\nabla F_{\ell} \neq \nabla E_{\ell}$ in Section 4, and show that this generalization results in the possibility of pattern forming instabilities even if there are only two species.

Motivated by Kurowski et al (2017), we consider solutions on spatially heterogeneous domains due to spatial resources and hazards in Section 5. We focus on the interplay between Turing patterns and these spatially heterogeneous domains by considering three-species models with parameters corresponding to diffusion driven instabilities of Section 3, which is distinct from the work in Kurowski et al (2017). When the spatial heterogeneity is small, we obtain Turing patterns which are modulated due to the spatial forcing. When the spatial heterogeneity is large, the Turing patterns may be destroyed, with distinct patterns emerging.

For each of these cases and behaviors, we outline the theoretical mechanism, and then provide explicit illustrative examples by way of numerical simulations. Our results therefore demonstrate a variety of routes to colony formation in models of the form (6), and classify which kinds of communities can admit pattern-forming instabilities in these systems.

\section{Colony formation due to diffusion and directed advection along fecundity gradients}

We study diffusion-driven instabilities leading to pattern formation through the Turing mechanism for the dimensionless model (6). In this section, we choose $b_{i j}=a_{i j}$ so that $\nabla F_{\ell}=\nabla E_{\ell}$.

\subsection{Turing instability framework}

Let $\mathbf{u}^{*}$ denote a spatially homogeneous positive (coexistence) steady state of (6a), that is, $E_{\ell}\left(\mathbf{u}^{*}\right)=0$ for all $\ell=1,2, \ldots, n$. Due to the spatial uniformity, the corresponding $\mathbf{w}_{\ell}^{*}=\mathbf{0}$ for all $\ell=1,2, \ldots, n$. We shall always assume that our choice of $\mathbf{u}^{*}$ is locally stable in the absence of diffusion. We note that steady states involving extinction of one or more species are also possible, but Turing instabilities from such states will either reduce to a model involving a smaller number of species, or lead to non-physical negative population densities. Therefore, we concentrate only on coexistence steady states.

We introduce a small perturbation about such a steady state, setting

$$
\begin{aligned}
\mathbf{u}(\mathbf{x}, t) & =\mathbf{u}^{*}+\sigma \tilde{\mathbf{u}} \exp (\lambda t+\mathrm{ik} \cdot \mathbf{x}), \\
\mathbf{w}_{\ell}(\mathbf{x}, t) & =\sigma \tilde{\mathbf{w}}_{\ell} \exp (\lambda t+\mathrm{i} \mathbf{k} \cdot \mathbf{x}),
\end{aligned}
$$

where $|\sigma| \ll 1$, $\tilde{\mathbf{u}}$ and $\tilde{\mathbf{w}}_{\ell}$ are constant vectors, $\mathbf{k} \in \mathbb{R}^{m}$ is a constant wavenumber vector, and $\lambda \in \mathbb{C}$.

Noting $\operatorname{grad}_{\mathbf{x}}(\hat{\mathbf{u}})$ becomes a matrix with $j$ th column $\left[i \tilde{u}_{j} \mathbf{k}\right] \exp (\lambda t+\mathrm{ik} \cdot \mathbf{x})$, we see after cancelling by a factor of $\exp (\lambda t+\mathrm{ik} \cdot \mathbf{x})$ that:

$$
\begin{gathered}
\lambda \tilde{u}_{i}=-\delta_{i}|\mathbf{k}|^{2} \tilde{u}_{i}-\mu_{i} u_{i}^{*} \mathrm{i}\left(\mathbf{k} \cdot \tilde{\mathbf{w}}_{i}\right)+\tilde{u}_{i}\left(\left.E_{i}\right|_{\mathbf{u}=\mathbf{u}^{*}}\right)+\left.u_{i}^{*} \tilde{\mathbf{u}} \cdot\left(\nabla_{\mathbf{u}} E_{i}\right)\right|_{\mathbf{u}=\mathbf{u}^{*}}, \\
\left(1+\epsilon_{i}|\mathbf{k}|^{2}\right) \tilde{\mathbf{w}}_{i}=\mathrm{ik}\left(\left.\tilde{\mathbf{u}} \cdot \nabla_{\mathbf{u}} E_{i}\right|_{\mathbf{u}=\mathbf{u}^{*}}\right),
\end{gathered}
$$

and we find

$$
\tilde{\mathbf{w}}_{i}=\frac{\mathrm{i}\left(\tilde{\mathbf{u}} \cdot\left(\left.\nabla_{\mathbf{u}} E_{i}\right|_{\mathbf{u}=\mathbf{u}^{*}}\right)\right)}{1+\epsilon_{i}|\mathbf{k}|^{2}} \mathbf{k}
$$

Equation (12a) becomes:

$$
\lambda \tilde{u}_{i}=-\delta_{i}|\mathbf{k}|^{2} \tilde{u}_{i}+\mu_{i} u_{i}^{*} \frac{\left(\tilde{\mathbf{u}} \cdot\left(\left.\nabla_{\mathbf{u}} E_{i}\right|_{\mathbf{u}=\mathbf{u}^{*}}\right)\right)}{1+\epsilon_{i}|\mathbf{k}|^{2}}|\mathbf{k}|^{2}+\tilde{u}_{i}\left(\left.E_{i}\right|_{\mathbf{u}=\mathbf{u}^{*}}\right)+\left.u_{i}^{*} \tilde{\mathbf{u}} \cdot\left(\nabla_{\mathbf{u}} E_{i}\right)\right|_{\mathbf{u}=\mathbf{u}^{*}},
$$

and the resulting linear system may be written in the form

$$
(\lambda \mathbf{I}-\mathbf{Q}) \tilde{\mathbf{u}}=0 .
$$


Here

$$
\mathbf{Q}\left(|\mathbf{k}|^{2}, \boldsymbol{\mu}\right)=\mathbf{R}-|\mathbf{k}|^{2} \mathbf{D}+\mathbf{M}
$$

is an $n \times n$ matrix consisting of

$$
\begin{gathered}
\mathbf{R}\left(\mathbf{u}^{*}\right)=-\left[u_{i}^{*} a_{i j}\right]_{n \times n}, \\
\mathbf{D}=\operatorname{diag}\left(\delta_{1}, \ldots, \delta_{n}\right) \\
\mathbf{M}\left(|\mathbf{k}|^{2}, \boldsymbol{\mu}\right)=-\left[m_{i} a_{i j}\right]_{n \times n}, \quad \text { where } \quad m_{i}\left(|\mathbf{k}|^{2}, \boldsymbol{\mu}\right)=\frac{\mu_{i} u_{i}^{*}|\mathbf{k}|^{2}}{1+\epsilon_{i}|\mathbf{k}|^{2}} .
\end{gathered}
$$

Note that $m_{i}(0, \boldsymbol{\mu})=0$ and $m_{i}\left(|\mathbf{k}|^{2}, \boldsymbol{\mu}\right) \geq 0$ for all $i \geq 1$. For convenience, we will at times make use of

$$
\mathbf{J}\left(\mathbf{u}^{*}\right)=\left[\frac{\partial E_{i}\left(\mathbf{u}^{*}\right)}{\partial u_{j}}\right]_{n \times n}=-\left[a_{i j}\right]_{n \times n},
$$

We are looking for a general perturbation where $\tilde{\mathbf{u}} \neq 0$, so we require $\operatorname{det}(\mathbf{Q}-\lambda \mathbf{I})=0$. We use this to find $\lambda_{1}\left(|\mathbf{k}|^{2}\right), \ldots, \lambda_{n}\left(|\mathbf{k}|^{2}\right) \in \mathbb{C}$. $\mathbf{M}$ relates to advection, whilst $\mathbf{R}$ corresponds to the reaction terms and $\mathbf{D}$ corresponds to diffusion. Note, for a Turing instability, we require a uniform steady state that is stable without diffusion or advection terms included, but can be driven unstable when diffusion is non-zero. This can be tested by requiring stability for $|\mathbf{k}|^{2}=0$, and instability for some $|\mathbf{k}|^{2}>0$.

In the cases where such a perturbation exists, we find that the homogeneous steady state is driven unstable for certain values of $\mathbf{k}$ that will depend on the domain. The existence of such wavenumbers does not determine the resulting pattern; once we move away from the uniform steady state, the non-linear dynamics takes over and determines the solution. Still, such a linear analysis is useful for identifying parameter regimes for which patterning may occur, and we use numerical simulations to later verify this. For simpler systems of reaction-diffusion equations, one can formally prove the existence of patterned states near to the Turing bifurcation, but we do not pursue this here. See Murray (2003) for a much more detailed discussion.

We shall make use of the Routh-Hurwitz criterion, which is as follows:

Theorem 1 (Routh-Hurwitz criterion) Consider the polynomial

$$
\chi(\lambda)=\lambda^{n}+a_{1} \lambda^{n-1}+\cdots+a_{n-1} \lambda+a_{n},
$$

where the coefficients $a_{i}$ are real constants for $i=1, \ldots, n$. Define the Hurwitz matrices

$$
\mathbf{H}_{1}=\left(a_{1}\right), \quad \mathbf{H}_{2}=\left(\begin{array}{cc}
a_{1} & 1 \\
a_{3} & a_{2}
\end{array}\right), \quad \ldots, \quad \mathbf{H}_{n}=\left(\begin{array}{cccccc}
a_{1} & 1 & 0 & 0 & \ldots & 0 \\
a_{3} & a_{2} & a_{1} & 1 & \ldots & 0 \\
a_{5} & a_{4} & a_{3} & a_{2} & \ldots & 0 \\
\vdots & \vdots & \vdots & \vdots & \ldots & \vdots \\
0 & 0 & 0 & 0 & \ldots & a_{n}
\end{array}\right)
$$

where $a_{i}=0$ for all $i>n$. Then all roots of the polynomial $p(\lambda)$ have negative real part if and only if

$$
\operatorname{det}\left(\mathbf{H}_{i}\right)>0, i=1,2, \ldots, n \text {. }
$$

If we consider the Hurwitz matrices resulting from the characteristic polynomial $\chi(\lambda)=\operatorname{det}(\lambda \mathbf{I}-$ Q) for the $n$-species model, then we obtain $n$ conditions for stability of each state. The characteristic polynomial reads

$$
\chi(\lambda)=\operatorname{det}(\lambda \mathbf{I}-\mathbf{Q})=\lambda^{n}-\operatorname{tr}(\mathbf{Q}) \lambda^{n-1}+\cdots+(-1)^{n} \operatorname{det}(\mathbf{Q}) .
$$

Applying Theorem 1, we find

$$
\begin{gathered}
\operatorname{tr}(\mathbf{Q})<0, \\
(-1)^{n} \operatorname{det}(\mathbf{Q})>0,
\end{gathered}
$$

for stability, along with $n-2$ more conditions depending on the coefficients of the polynomial $p$.

We shall always assume that the spatially uniform state $\mathbf{u}^{*}$ satisfies the Routh-Hurwitz criterion in the absence of diffusion $(|\mathbf{k}|=0)$. Then, to find a diffusive instability, it is necessary to identify parameter spaces for which one of the Routh-Hurwitz criteria fail given $\mathbf{k}$ satisfying $0<|\mathbf{k}|<\infty$. 


\subsection{No diffusive instability for two species with advection toward fecundity}

We first consider the two-species form of the model. For stable reaction kinetics at the coexistence steady state $\mathbf{u}^{*}$, we require $\operatorname{tr}(\mathbf{R})=-\left(a_{11} u_{1}^{*}+a_{22} u_{2}^{*}\right)<0$ and $\operatorname{det}(\mathbf{J})>0$. From the definition $(16)$ and the conditions (25), we have for $\mu_{1}, \mu_{2} \geq 0$ that

$$
\operatorname{tr}(\mathbf{Q})=\operatorname{tr}(\mathbf{R})-\left(\delta_{1}+\delta_{2}\right)|\mathbf{k}|^{2}-\frac{a_{11} \mu_{1} u_{1}^{*}|\mathbf{k}|^{2}}{1+\epsilon_{1}|\mathbf{k}|^{2}}-\frac{a_{22} \mu_{2} u_{2}^{*}|\mathbf{k}|^{2}}{1+\epsilon_{2}|\mathbf{k}|^{2}}<0
$$

and

$$
\begin{aligned}
\operatorname{det}(\mathbf{Q})= & u_{1}^{*} u_{2}^{*} \operatorname{det}(\mathbf{J})+\delta_{1} \delta_{2}|\mathbf{k}|^{4}+\left\{\delta_{1} a_{22} u_{2}^{*}+\delta_{2} a_{11} u_{1}^{*}+\frac{\delta_{1} a_{22} \mu_{2} u_{2}^{*}|\mathbf{k}|^{2}}{1+\epsilon_{2}|\mathbf{k}|^{2}}+\frac{\delta_{2} a_{11} \mu_{1} u_{1}^{*}|\mathbf{k}|^{2}}{1+\epsilon_{1}|\mathbf{k}|^{2}}\right\}|\mathbf{k}|^{2} \\
& +u_{1}^{*} u_{2}^{*} \operatorname{det}(\mathbf{J})\left\{\frac{\mu_{1} \mu_{2}|\mathbf{k}|^{4}}{\left(1+\epsilon_{1}|\mathbf{k}|^{2}\right)\left(1+\epsilon_{2}|\mathbf{k}|^{2}\right)}+\frac{\mu_{1}|\mathbf{k}|^{2}}{1+\epsilon_{1}|\mathbf{k}|^{2}}+\frac{\mu_{2}|\mathbf{k}|^{2}}{1+\epsilon_{2}|\mathbf{k}|^{2}}\right\} \\
> & 0,
\end{aligned}
$$

since $a_{j j}>0$ for each $j$. This implies that any general spatial perturbation of the form (11) is stable when $n=2$ and $\nabla F_{\ell}=\nabla E_{\ell}$. Therefore, in the case of two species models, diffusion driven instability through the Turing mechanism is not possible when the resource gradient is in the direction of fecundity, as shown on two-dimensional domains in Kurowski et al (2017) under the assumption of irrotational vector fields $\mathbf{w}_{\ell}$ in the plane. Hence, our results generalize this finding to arbitrary domains in $\mathbb{R}^{m}$ without the need for the irrotational assumption.

That there are no diffusive instabilities in the case of two species is not surprising, as it has been shown that a variety of commonly used predator-prey kinetics do not admit the Turing instability when they are spatially extended (Fasani and Rinaldi, 2011), since they are not of the activator-inhibitor form. What we have shown is that, when the directed motion is along a gradient corresponding to fecundity, these findings will not change. In Section 4 we shall demonstrate that when $\nabla F_{\ell} \neq \nabla E_{\ell}$ there may be pattern formation in the case of $n=2$ species, due to the advection direction differing from the fecundity gradient.

\subsection{Diffusive instability for three or more species}

Although diffusive instabilities will not occur for two-species models with $\nabla F_{\ell}=\nabla E_{\ell}$, one may show that they can occur for three or more species. We therefore focus on the three-species case, as this demonstrates the useful dynamics without becoming too cumbersome analytically. This extends existing results for $n$-species type reaction-diffusion models (Dillon et al, 1994; Satnoianu et al, 2000) to the class of models (6) with advection toward resource gradients. In the scenario where only one or two species survive at steady state, the results reduce to those for one or two species models, and spatial pattern formation does not occur for $\nabla F_{\ell}=\nabla E_{\ell}$. Therefore, we shall restrict our attention to dynamics where all three populations persist at steady state, that is $u_{\ell}^{*}>0$ for $\ell=1,2,3$.

At a stable coexistence steady state, $\operatorname{det}(\mathbf{J})<0$, and hence $\mathbf{J}$ is invertible. The unique steady state may then be represented by $\mathbf{u}^{*}=-\mathbf{J}^{-1} \mathbf{A}$, hence

$$
\mathbf{u}^{*}=\frac{-1}{\operatorname{det}(\mathbf{J})}\left(\begin{array}{ccc}
\mathcal{J}_{11} & -\mathcal{J}_{12} & \mathcal{J}_{13} \\
-\mathcal{J}_{21} & \mathcal{J}_{22} & -\mathcal{J}_{23} \\
\mathcal{J}_{31} & -\mathcal{J}_{32} & \mathcal{J}_{33}
\end{array}\right)\left(\begin{array}{l}
A_{1} \\
A_{2} \\
A_{3}
\end{array}\right)
$$

Here we use the notation $\mathcal{J}_{i j}$ for the $(i, j)$ th minor of the matrix $\mathbf{J}$, which is found by removing the $i$ th row and $j$ th column from $\mathbf{J}$ and then taking the determinant of the resulting $2 \times 2$ matrix. For instance we have the three principal minors $\mathcal{J}_{11}=a_{22} a_{33}-a_{23} a_{32}, \mathcal{J}_{22}=a_{11} a_{33}-a_{13} a_{31}$, and $\mathcal{J}_{33}=a_{11} a_{22}-a_{12} a_{21}$. Since $u_{\ell}^{*}>0$ for all $\ell=1,2,3$, we obtain the constraint

$$
\sum_{j=1}^{3}(-1)^{i+j} \mathcal{J}_{i j} A_{j}>0
$$

for all $i$, since $\operatorname{det}(\mathbf{J})<0$. 
When there are $n=3$ species, we find that (16) reduces to

$$
\mathbf{Q}\left(|\mathbf{k}|^{2}, \boldsymbol{\mu}\right)=\operatorname{diag}\left(u_{1}^{*}+m_{1}, u_{2}^{*}+m_{2}, u_{3}^{*}+m_{3}\right) \mathbf{J}\left(\mathbf{u}^{*}\right)-|\mathbf{k}|^{2} \mathbf{D},
$$

and the characteristic polynomial becomes

$$
\operatorname{det}(\lambda \mathbf{I}-\mathbf{Q})=\lambda^{3}-\alpha_{1} \lambda^{2}+\alpha_{2} \lambda-\alpha_{3},
$$

where

$$
\begin{gathered}
\alpha_{1}=\operatorname{tr}(\mathbf{Q}), \\
\alpha_{2}=\left(Q_{11} Q_{22}+Q_{11} Q_{33}+Q_{22} Q_{33}\right)-\left(Q_{12} Q_{21}+Q_{13} Q_{31}+Q_{23} Q_{32}\right), \\
\alpha_{3}=\operatorname{det}(\mathbf{Q}) .
\end{gathered}
$$

Applying the Routh-Hurwitz Criterion, the matrix (30) is stable provided $\alpha_{1}<0, \alpha_{3}<0$, and $\alpha_{1} \alpha_{2}-$ $\alpha_{3}<0$. This is equivalent to the three conditions

$$
\begin{gathered}
\operatorname{tr}(\mathbf{Q})<0 \\
\operatorname{det}(\mathbf{Q})<0 \\
\operatorname{tr}(\mathbf{Q})\left[\left(Q_{11} Q_{22}+Q_{11} Q_{33}+Q_{22} Q_{33}\right)-\left(Q_{12} Q_{21}+Q_{13} Q_{31}+Q_{23} Q_{32}\right)\right]-\operatorname{det}(\mathbf{Q})<0 .
\end{gathered}
$$

If we have stability when $|\mathbf{k}|^{2}=0$, but allow $|\mathbf{k}|^{2}>0$ to change the sign of any of these conditions, then we have a diffusion driven instability. We shall assume stable reaction kinetics at the spatially uniform steady state, and hence that (33) always holds for $|\mathbf{k}|^{2}=0$.

Since we are considering the case of logistic growth of each individual population, $a_{j j}>0$, one may show that (33a) holds for all $\mathbf{k}$,

$$
\operatorname{tr}\left(\left(\mathbf{Q}\left(|\mathbf{k}|^{2}, \boldsymbol{\mu}\right)\right)=-\sum_{j=1}^{3}\left(u_{j}^{*}+m_{j}\right) a_{j j}-\left(\delta_{1}+\delta_{2}+\delta_{3}\right)|\mathbf{k}|^{2} \leq \operatorname{tr}(\mathbf{Q}(0,0))<0,\right.
$$

as is common for the Turing instability. Therefore, only conditions (33b) or (33c) may change sign with diffusion leading to an instability.

Regarding condition (33b), using (30) we find

$$
\begin{aligned}
\operatorname{det}\left(\mathbf{Q}\left(|\mathbf{k}|^{2}, \boldsymbol{\mu}\right)\right)= & -\delta_{1} \delta_{2} \delta_{3}|\mathbf{k}|^{6}-\left(\delta_{2} \delta_{3} a_{11}\left(u_{1}^{*}+m_{1}\right)+\delta_{1} \delta_{3} a_{22}\left(u_{2}^{*}+m_{2}\right)+\delta_{1} \delta_{2} a_{33}\left(u_{3}^{*}+m_{3}\right)\right)|\mathbf{k}|^{4} \\
& -\left(\delta_{1} \mathcal{J}_{11}\left(u_{2}^{*}+m_{2}\right)\left(u_{3}^{*}+m_{3}\right)+\delta_{2} \mathcal{J}_{22}\left(u_{1}^{*}+m_{1}\right)\left(u_{3}^{*}+m_{3}\right)\right. \\
& \left.\quad+\delta_{3} \mathcal{J}_{33}\left(u_{1}^{*}+m_{1}\right)\left(u_{2}^{*}+m_{2}\right)\right)|\mathbf{k}|^{2} \\
& +\left(u_{1}^{*}+m_{1}\right)\left(u_{2}^{*}+m_{2}\right)\left(u_{3}^{*}+m_{3}\right) \operatorname{det}(\mathbf{J})
\end{aligned}
$$

Note that from (19) each $m_{i}$ is a rational function in $|\mathbf{k}|$ and hence so is the determinant (35), in contrast to a polynomial in $|\mathbf{k}|$ as commonly found in the context of the Turing instability. Therefore, the analysis involved in signing (35) is more complicated than that of a polynomial of degree three in $|\mathbf{k}|^{2}$. We observe that the coefficients of the $|\mathbf{k}|^{6}$ and $|\mathbf{k}|^{4}$ terms are strictly negative (since $\delta_{j}>0$ ), while the term involving $\operatorname{det}(\mathbf{J})$ is also negative (since $\operatorname{det}(\mathbf{J})<0$ for stable reaction kinetics). Therefore, in order to have an instability result from $\operatorname{det}\left(\mathbf{Q}\left(|\mathbf{k}|^{2}, \boldsymbol{\mu}\right)\right)>0$, a necessary condition is that

$$
\delta_{1} \mathcal{J}_{11}\left(u_{2}^{*}+m_{2}\right)\left(u_{3}^{*}+m_{3}\right)+\delta_{2} \mathcal{J}_{22}\left(u_{1}^{*}+m_{1}\right)\left(u_{3}^{*}+m_{3}\right)+\delta_{3} \mathcal{J}_{33}\left(u_{1}^{*}+m_{1}\right)\left(u_{2}^{*}+m_{2}\right)<0,
$$

since the coefficient of $|\mathbf{k}|^{2}$ is the only term which has the possibility of being positive and large enough to force $\operatorname{det}\left(\mathbf{Q}\left(|\mathbf{k}|^{2}, \boldsymbol{\mu}\right)\right)>0$, as well. However, (36) will not hold if $\mathcal{J}_{j j}>0$ for all $j=1,2,3$, and this rules out a variety of reaction kinetics (as we shall later discuss).

Obtaining sufficient conditions for $\operatorname{det}\left(\mathbf{Q}\left(|\mathbf{k}|^{2}, \boldsymbol{\mu}\right)\right)>0$ is challenging, since (35) is a rational function in $|\mathbf{k}|^{2}$. This rational function may be put into the form of a polynomial in $|\mathbf{k}|^{2}$, however it will be of degree six in the variable $|\mathbf{k}|^{2}$, which is not in general solvable with analytical methods. As such, we shall investigate parameter sets satisfying the necessary condition (36) and then test these parameter values numerically to see that they indeed do permit $\operatorname{det}\left(\mathbf{Q}\left(|\mathbf{k}|^{2}, \boldsymbol{\mu}\right)\right)>0$. 
Regarding condition (33c), note that we can phrase this condition in terms of $|\mathbf{k}|$, akin to what we have shown in (35). The resulting condition is even more complicated than that given in (35), and does not provide any additional intuition, so we omit it. In practice, we test this or (33c) directly with fixed parameter values in the case where we consider an instability arising from (33c) changing sign.

Recalling $a_{j j}>0$, we can classify all possible reaction schemes by categorising $a_{21}, a_{31}$, and $a_{32}$ as positive, negative, or zero. We then label the species 1,2 , and 3 such that if $i>j$, then species $i$ is either on the same trophic level or a higher trophic level than species $j$. This in turn allows us to determine whether $a_{12}, a_{13}$, and $a_{23}$ are positive, negative, or zero. We have three categorisations for three parameters, leading to twenty seven cases, but we find fifteen mathematically distinct cases by ignoring cases that are equivalent down to a relabelling of the species. The cases vary depending on which species the predators feed on, and whether species without a predator-prey interaction compete for resources, or do not interact at all. We partition these further into the cases where there are one, two, or three herbivores.

We now consider the possible trophic interactions which can admit pattern-forming instabilities. These can all be understood via the signs of the community or interaction matrices (note that the terminology differs based on quantitative descriptions of the interactions, but that the sign of $\mathbf{J}$ will be the same as the signs of these different representations; see (Novak et al, 2016)). In the case where we have one herbivore (species 1) and the remaining species are either omnivores (intraguild predators) or carnivores (predators), we find the matrix $-\mathbf{J}$ can take one of the following five possible forms:

$$
\begin{aligned}
& \left(\begin{array}{c}
++0 \\
-++ \\
0-+
\end{array}\right),(37 \mathrm{a}) \quad\left(\begin{array}{c}
+++ \\
-++ \\
-++
\end{array}\right),(37 \mathrm{~b})\left(\begin{array}{c}
+++ \\
-+0 \\
-0+
\end{array}\right) \\
& \left(\begin{array}{c}
+++ \\
-++ \\
-++
\end{array}\right),(37 \mathrm{~d}) \quad\left(\begin{array}{c}
+++ \\
-++ \\
+-+
\end{array}\right) .(37 \mathrm{e})
\end{aligned}
$$

In cases $(37 \mathrm{a}),(37 \mathrm{~b})$, and $(37 \mathrm{c})$ we find $\mathcal{J}_{j j}>0$ for all $j$, and hence these cannot result in condition (33b) changing sign due to an instability which causes $\operatorname{det}\left(\mathbf{Q}\left(|\mathbf{k}|^{2}, \boldsymbol{\mu}\right)\right)>0$. This is not true of cases $(37 \mathrm{~d})$ and $(37 \mathrm{e})$, and we find both may support diffusive instabilities, depending on the parameter choices. Some of these are well-known community structures, such as (37a) which represents a tritrophic food chain, and $(37 \mathrm{~d})$ which represents two predators competing for the same prey (and possibly other resources), while others such as (37e) are less common. Such interactions are unlikely in isolated communities, but can occur in large food webs, especially due to weak interactions (Zhao et al, 2019).

In the case where we have two herbivores (species 1 and 2) and the remaining species is either omnivorous or carnivorous, we have the following six possible forms for the matrix $-\mathbf{J}$ :

$$
\begin{aligned}
& \left(\begin{array}{c}
+0+ \\
0++ \\
--+
\end{array}\right), \quad(38 \mathrm{a})\left(\begin{array}{c}
+0 \\
0 \\
0+ \\
0-+
\end{array}\right),(38 \mathrm{~b})\left(\begin{array}{c}
+++ \\
+++ \\
--+
\end{array}\right) \\
& \left(\begin{array}{c}
++0 \\
+++ \\
0-+
\end{array}\right),(38 \mathrm{~d}) \quad\left(\begin{array}{c}
+++ \\
+++ \\
+-+
\end{array}\right),(38 \mathrm{e})\left(\begin{array}{c}
+0+ \\
0++ \\
+-+
\end{array}\right) .
\end{aligned}
$$

Considering the form of the matrices given by case (38a) and (38b), we find $\mathcal{J}_{i i}>0$ for all $j$. However, this is not true of cases $(38 \mathrm{c}),(38 \mathrm{~d}),(38 \mathrm{e})$ and $(38 \mathrm{f})$; we find that all four of these cases can lead to diffusive instabilities through a change in sign of condition (33b) for some parameters.

In the case where we have three herbivores, we have the following four possible forms for the matrix $-\mathbf{J}$ :

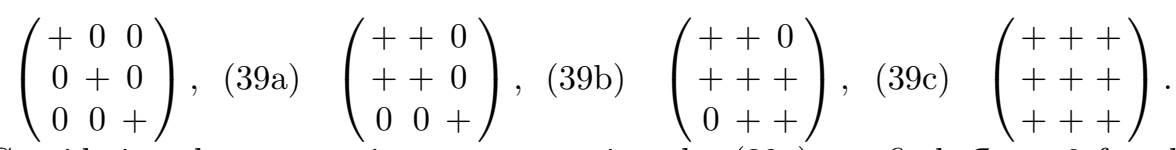

Considering the community structures given by (39a), we find $\mathcal{J}_{j j}>0$ for all $j$. In (39b), we find $\mathcal{J}_{11}, \mathcal{J}_{22}>0$, but the sign of $\mathcal{J}_{33}$ depends on the strength of interactions. However, for stability of the reaction kinetics in the absence of diffusion we must have $\operatorname{det}(\mathbf{J})=-a_{33} J_{33}<0$, and hence must have $\mathcal{J}_{33}>0$. So we have $\mathcal{J}_{j j}>0$ for all $j$. In (39c), we find $\mathcal{J}_{22}>0$, but we do not know the sign of $\mathcal{J}_{11}$ or $\mathcal{J}_{33}$. However, we have $\operatorname{det}(\mathbf{J})=-a_{11} \mathcal{J}_{11}+a_{12} \mathcal{J}_{12}=a_{32} \mathcal{J}_{32}-a_{33} \mathcal{J}_{33}$. Since $a_{12} \mathcal{J}_{12}>0$ and $a_{32} \mathcal{J}_{32}>0$, 
we must have that $\mathcal{J}_{11}>0$ and $\mathcal{J}_{33}>0$ so that $\operatorname{det}(\mathbf{J})<0$. Again we have $\mathcal{J}_{j j}>0$ for all $j$. We therefore find that $(39 \mathrm{a}),(39 \mathrm{~b})$, and (39c) cannot support diffusive instabilities through a change in sign of condition $(33 \mathrm{~b})$. This is not true of case $(39 \mathrm{~d})$; this case can lead to diffusive instabilities through a change in sign of condition $(33 \mathrm{~b})$.

Having classified which forms of the reaction kinetics may permit diffusive instabilities and spatial patterning through the Turing mechanism, we shall now choose representative cases to simulate. We use MATLAB to determine whether each system has a positive stable steady state. To fully explore the behavior of the model, we find parameter combinations where we will get diffusive instabilities caused by either condition (33b) or condition (33c) changing signs. We sweep the parameter space by varying $a_{i j}$ and $A_{j}$, searching for combinations that result in positive stable steady states. We do not allow cases where $\mathcal{J}_{j j} \geq 0$ for all $j$, and hence we consider cases corresponding to (37d), (37e), (38c), (38d), (38e), $(38 \mathrm{f})$, and $(39 \mathrm{~d})$. In each case we find some parameters for which diffusion-driven instabilities occur, though due to the size of the parameter space we do not fully classify which of these lead to stationary patterns (representing species aggregating in colonies or niches but coexisting across the whole domain) or which lead to spatiotemporal behavior.

We use the commercially-available finite element software COMSOL to simulate the dimensionless PDE system (6), and the dimensionless domain is taken to be the unit square, $\Omega=[0,1]^{2}$ in the cases discussed. The initial condition is taken to be the positive spatially uniform steady state found using MATLAB, plus a small random perturbation. This perturbation is generated by adding a normal random variable with mean zero and standard deviation of $10^{-2}$ to each discrete element. The figures in this section were generated using a mesh containing 6282 domain elements and 200 boundary elements, and convergence for each simulation was checked by considering finer meshes.

In Figure 1 we consider the case (38e) which results in condition (33b) changing sign. Such a community involves two competing species, where one of them is preyed upon by a third species. For instance, if there is a large size difference between the herbivorous species such that only one experiences predation, or if both are preyed upon but one of the prey has sufficient defenses to make predation harmful for both prey and predator (e.g. poison). We see that the second species, which is preyed upon, only has a small spatial variation across the domain, whereas the predator and the competing herbivore segregate into distinct niches. The structure of these regions depends heavily on the direction motion, despite the advection coefficients being much smaller than the interaction coefficients. This result suggests that considering advection is crucial to the model prediction, since incorporating rational movement along resource gradients as opposed to purely random movement has a large impact on the structure of niches for some species. An enhanced rate of advection appears to enhance the degree of segregation between competing species, with increasingly localized patterns emerging for increasing rates of advection $\mu_{j}$. We remark as well that these choices parameters correspond to differential advection, so it is not surprising that this additional differential transport can give some robustness and variation to the observed patterns.

In Figure 2 we provide an example of community interactions of the form (38c) which results in the change of sign of condition (33c). Such a community represents two competing species both being consumed by a predator. For this parameter set, the predator exists with a natural decay rate in the absence of prey, as $A_{3}<0$. It therefore requires sufficient prey to be available or it will face extinction. For these parameters, we observe spatiotemporal patterns, with colonies forming and moving around the domain in an oscillatory manner, with the predator populations chasing the other species. Such spatiotemporal oscillations have been predicted in a range of theoretical models, and their correspondence with ecological reality is of current interest (Sherratt et al, 1997; Baurmann et al, 2007; Malchow et al, 2007; Bennett and Sherratt, 2019). We should, however, remark that spatiotemporal patterns suggesting colony motion are not generic to the case where an instability results from (33c) changing sign. Interestingly, if the directed motion is absent or uniform between populations (e.g. $\mu_{\ell}=\mu_{j}$ for all $\ell, j$ ), then there is no instability and the system decays back to a stable coexistence state. However, if the diffusion coefficients are set equal between all three species, then there is still a spatiotemporal oscillation comparable to what is shown in this Figure. Hence these oscillations are, in this case, due to the presence of differential directed motion.

In Figure 3, we present another case where a diffusive instability results from (33c) changing sign, yet we observe stationary Turing-type patterns. For this example, we consider the dynamics of three species mutually competing for resources, corresponding to the community structure (39d). In this case, the first species is almost spatially homogeneous, coexisting alongside both of the others with a slight variation in its distribution due to the presence of the third species and the slightly stronger inter-species competition $a_{13}$. In contrast, the second and third species have almost entirely separated into distinct 
(a)

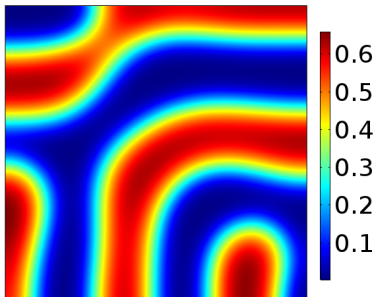

(b)

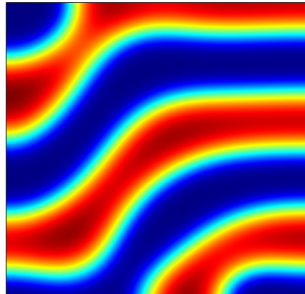

(c)

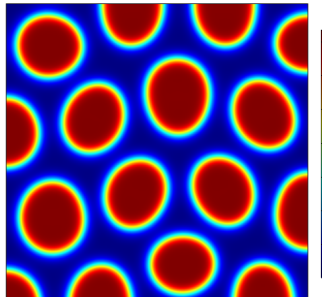

$\mathrm{u}_{1}$
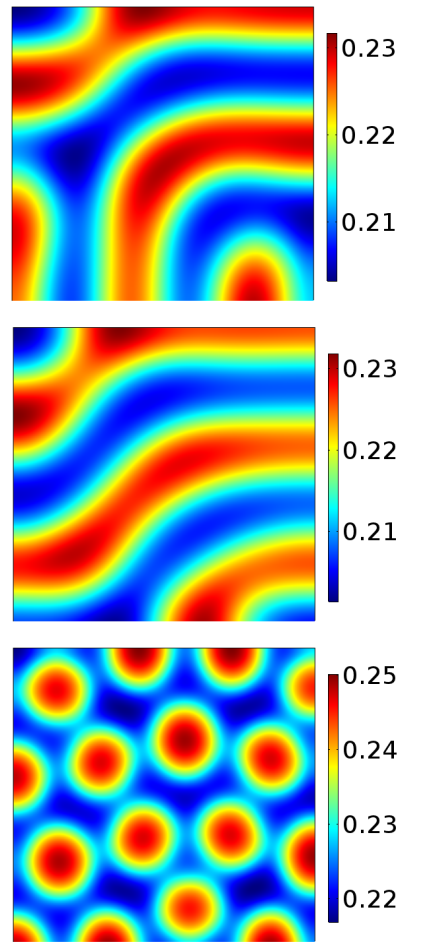

$\mathrm{u}_{2}$

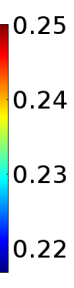

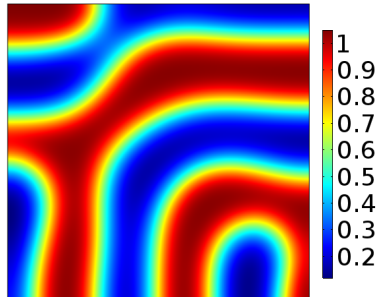
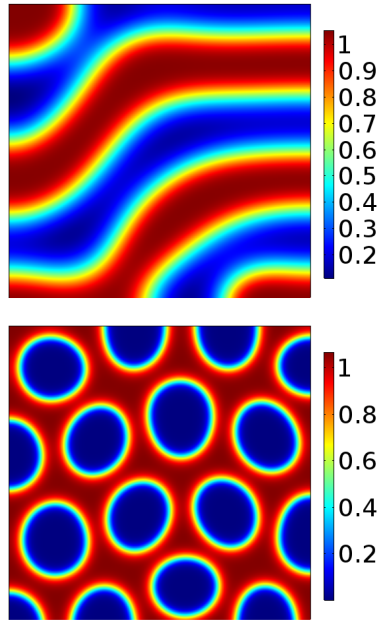

$\mathrm{u}_{3}$

Fig. 1 Simulations of (6) showing spatial patterning in generalist predator-prey dynamics, corresponding to (38e). The values used to produce these plots are given by the reaction kinetic parameters $a_{11}=1.09, a_{12}=0.8, a_{13}=0.81, a_{21}=0.82$, $a_{22}=1.1, a_{23}=0.83, a_{31}=1.68, a_{32}=-0.85, a_{33}=1.11$, and $A_{j}=1$ for $j=1,2,3$, while the diffusion parameters satisfy $\delta_{1}=10^{-5}, \delta_{2}=10^{-2}, \delta_{3}=10^{-4}$. Regarding the advection parameters, we choose $(\mathrm{a}) \boldsymbol{\mu}=\mathbf{0},(\mathrm{b}) \mu_{1}=10^{-3}$, $\mu_{2}=10^{-4}, \mu_{3}=10^{-4}$, (c) $\mu_{1}=10^{-3}, \mu_{2}=10^{-2}, \mu_{3}=10^{-1}$. In (b) we use $\left(\epsilon_{1}, \epsilon_{2}, \epsilon_{3}\right)=\left(10^{-3}, 10^{-2}, 10^{-1}\right)$ while in $(\mathrm{c})$ we use $\epsilon_{\ell}=10^{-2}$ for all $\ell=1,2,3$. We present simulations at time $t=35000$, by which point the patterns have stabilized.
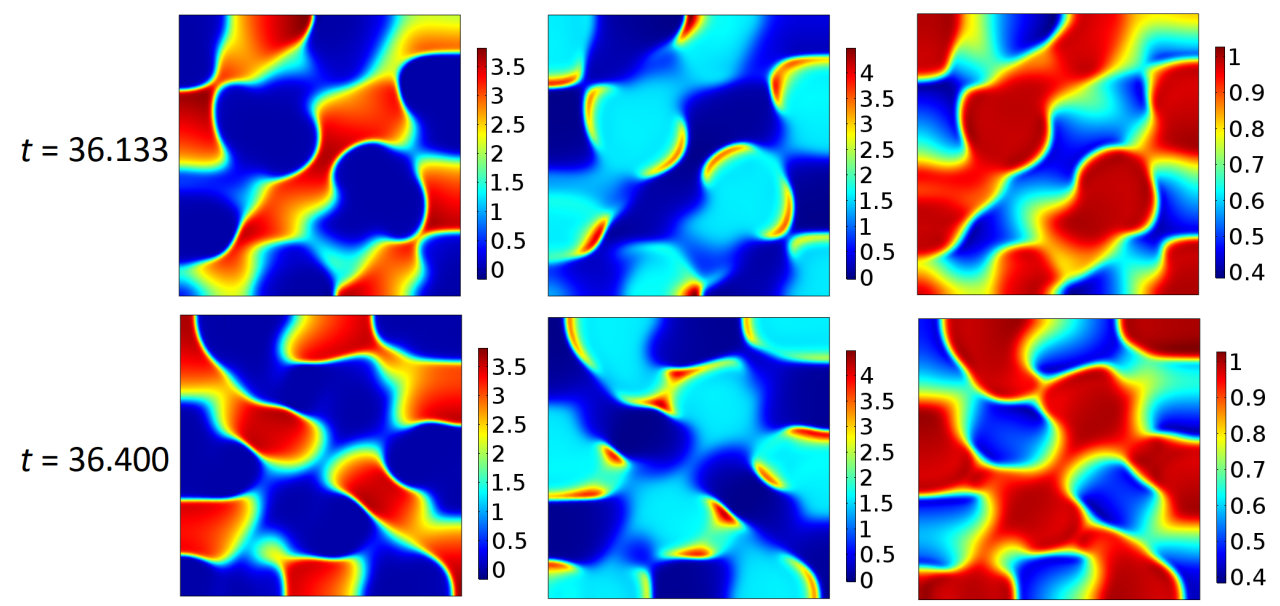

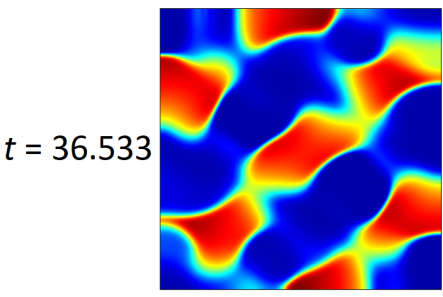

$\mathrm{u}_{1}$

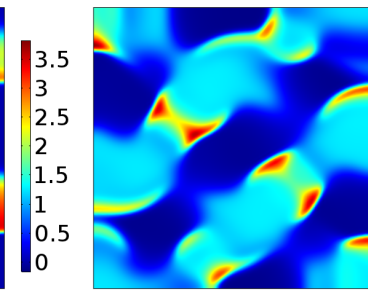

$\mathrm{u}_{2}$

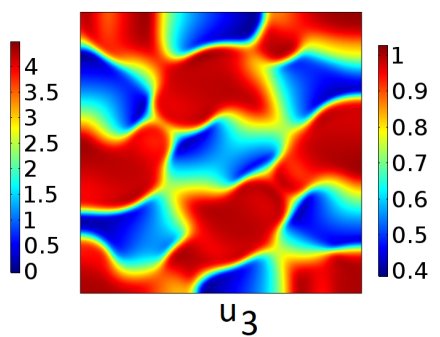

Fig. 2 Simulations of (6) showing spatiotemporal patterning in a generalist predator-prey system with one predator, corresponding to reaction kinetics of the form (38c). The values used to produce these plots are given by the reaction kinetic parameters $a_{11}=1.09, a_{12}=1.6, a_{13}=2.43, a_{21}=1.64, a_{22}=1.1, a_{23}=7.47, a_{31}=-1.68, a_{32}=-5.1$, $a_{33}=1.11, A_{1}=5, A_{2}=9, A_{3}=-7$, while the diffusion and advection parameters satisfy $\delta_{1}=\delta_{2}=10^{-2}, \delta_{3}=10^{-3}$, $\mu_{1}=0.8, \mu_{2}=0.4, \mu_{3}=10^{-2}, \epsilon_{\ell}=10^{-2}, \ell=1,2,3$. 


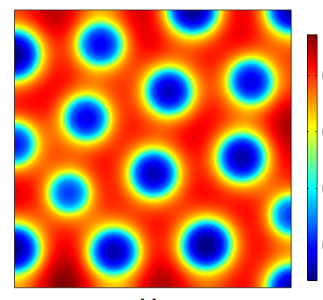

$\mathrm{u}_{1}$

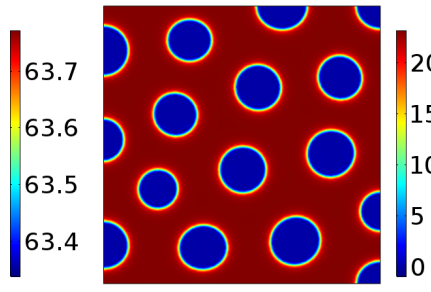

$\mathrm{u}_{2}$

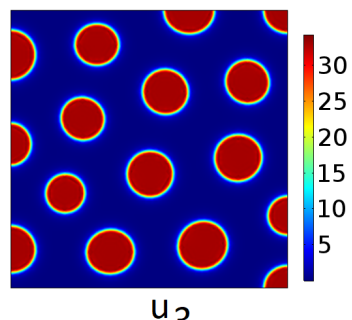

$u_{3}$

Fig. 3 Simulations of (6) showing spatial patterning resulting from competition dynamics taking the form of (39d). The values used to produce these plots are given by the reaction kinetic parameters $a_{11}=1.09, a_{12}=0.8, a_{13}=0.81$, $a_{21}=1.64, a_{22}=1.1, a_{23}=0.83, a_{31}=0.84, a_{32}=1.7, a_{33}=1.11, A_{1}=0.09, A_{2}=0.13, A_{3}=0.09$, while the diffusion and advection parameters satisfy $\delta_{1}=10^{-4}, \delta_{2}=10^{-6}, \delta_{3}=10^{-4}, \mu_{1}=10^{-3}, \mu_{2}=0.5, \mu_{3}=10^{-3}, \epsilon_{\ell}=10^{-4}, \ell=1,2,3$. Time is fixed at $t=10^{6}$.

spatial regions, in part due to the strong impact of $u_{2}$ on $u_{3}$ via $a_{32}$. Nevertheless, the slight variation in $u_{1}$ is necessary to mediate the segregation of the other two species, as if it were perfectly homogeneous then the dynamics would behave as a two-species model which cannot form spatial patterns. As this first population has reached a much higher density than the others, small variations in its distribution can profoundly influence the spatial organisation of the other two species.

\section{Colony formation due to directed advection which is not along fecundity gradients}

While advection toward resource gradients in the direction of fecundity, $\nabla F_{\ell}=\nabla E_{\ell}$, was considered in the previous section and on all prior published work on the model (1), in principle one may generalize this so that species move due to advection in another manner. For instance, in more realistic scenarios, species do not have perfect information, particularly across large distances, and might therefore try and approximate the optimal direction to move, and this guess is seldom likely to be the precise optimal direction in the sense of increasing fecundity, $\nabla E_{\ell}$. Indeed, many reaction-diffusion-advection models feature advection due to an external source (Bernasconi and Boissonade, 1997; Krause et al, 2018a; Míguez et al, 2006; Nakagaki et al, 1999; Nugent et al, 2004; Sherratt, 2011), such as motion induced by water or air flow (Andresén et al, 1999; Dawson et al, 1994; Rovinsky and Menzinger, 1992). The choice of advection which is linearly proportional to population density is simpler than other models of directed motion, such as those presented in Potts and Lewis (2019), but allows for a simple interpretation and analysis of the attraction or repulsion between populations. Additionally, it is the simplest generalization of the model (1) which leads to substantially different behavior.

In the present section, we relax the requirement $\nabla F_{\ell}=\nabla E_{\ell}$, and use more general $E_{\ell}$ and $F_{\ell}$ as defined in (6c)-(6d), with $b_{i j}$ not in general equal to $a_{i j}$. While one can consider a variety of scenarios, we focus on the fact that this generalization provides a new route to spatial patterning and colony formation in the model (6). To demonstrate this, we focus our attention on the dynamics of $n=2$ species. Whereas it was shown in Section 3.2 that the model with $\nabla F_{\ell}=\nabla E_{\ell}$ does not permit pattern formation due to the Turing mechanism when two species are considered, we shall show that when $\nabla F_{\ell} \neq \nabla E_{\ell}$ pattern formation does emerge in the case of two species. We shall also assume $\epsilon_{1}=\epsilon_{2}=\epsilon$ for simplicity.

We again consider spatial perturbations of the form (11). Using these spatial perturbations in (6b), we find that the constant vectors $\tilde{\mathbf{w}}_{\ell}$ must take the form

$$
\tilde{\mathbf{w}}_{\ell}=\frac{i|\mathbf{k}|^{2}}{1+\epsilon|\mathbf{k}|^{2}}\left(b_{\ell 1} \tilde{u}_{1}+b_{\ell 2} \tilde{u}_{2}\right) \text {. }
$$

Linearizing (6a) and using the spatial perturbations (11), we find that the stability matrix (30) takes the form

$$
\mathbf{Q}\left(|\mathbf{k}|^{2}, \boldsymbol{\mu}\right)=\left(\begin{array}{l}
-a_{11} u_{1}^{*}-a_{12} u_{1}^{*} \\
-a_{21} u_{2}^{*}-a_{22} u_{2}^{*}
\end{array}\right)-|\mathbf{k}|^{2}\left(\begin{array}{cc}
\delta_{1} & 0 \\
0 & \delta_{2}
\end{array}\right)-\frac{|\mathbf{k}|^{2}}{1+\epsilon|\mathbf{k}|^{2}}\left(\begin{array}{l}
b_{11} \mu_{1} u_{1}^{*} b_{12} \mu_{1} u_{1}^{*} \\
b_{21} \mu_{2} u_{2}^{*} b_{22} \mu_{2} u_{2}^{*}
\end{array}\right) .
$$

The corresponding characteristic polynomial for the temporal eigenvalues $\lambda$ reads

$$
\operatorname{det}(\lambda \mathbf{I}-\mathbf{Q})=\lambda^{2}-\operatorname{tr}\left(\mathbf{Q}\left(|\mathbf{k}|^{2}, \boldsymbol{\mu}\right)\right) \lambda+\operatorname{det}\left(\mathbf{Q}\left(|\mathbf{k}|^{2}, \boldsymbol{\mu}\right)\right)=0,
$$


where

$$
\operatorname{tr}\left(\mathbf{Q}\left(|\mathbf{k}|^{2}, \boldsymbol{\mu}\right)\right)=-\left(a_{11} u_{1}^{*}+a_{22} u_{2}^{*}\right)-\left(\delta_{1}+\delta_{2}\right)|\mathbf{k}|^{2}-\frac{\left(b_{11} \mu_{1} u_{1}^{*}+b_{22} \mu_{2} u_{2}^{*}\right)|\mathbf{k}|^{2}}{1+\epsilon|\mathbf{k}|^{2}}
$$

and

$$
\begin{aligned}
\operatorname{det}\left(\mathbf{Q}\left(|\mathbf{k}|^{2}, \boldsymbol{\mu}\right)\right)= & \left(a_{11} a_{22}-a_{12} a_{21}\right) u_{1}^{*} u_{2}^{*}+\delta_{1} \delta_{2}|\mathbf{k}|^{4}+\left(\delta_{1} a_{22} u_{2}^{*}+\delta_{2} a_{11} u_{1}^{*}\right)|\mathbf{k}|^{2} \\
& +\left(\delta_{1} b_{22} \mu_{2} u_{2}^{*}+\delta_{2} b_{11} \mu_{1} u_{1}^{*}\right) \frac{|\mathbf{k}|^{4}}{1+\epsilon|\mathbf{k}|^{2}}+\frac{\left(b_{11} b_{22}-b_{12} b_{21}\right) \mu_{1} \mu_{2} u_{1}^{*} u_{2}^{*}|\mathbf{k}|^{4}}{\left(1+\epsilon|\mathbf{k}|^{2}\right)^{2}} \\
& +\frac{\left\{\left(a_{22} b_{11}-a_{21} b_{12}\right) \mu_{1}+\left(a_{11} b_{22}-a_{12} b_{21}\right) \mu_{2}\right\} u_{1}^{*} u_{2}^{*}|\mathbf{k}|^{2}}{1+\epsilon|\mathbf{k}|^{2}} .
\end{aligned}
$$

There are two roots to (42), and a wavenumber vector $\mathbf{k}$ leads to a linear instability if $\operatorname{Re}(\lambda(\mathbf{k}))>0$ for at least one of the roots. By the Routh-Hurwitz stability criterion, there exists a root $\lambda(\mathbf{k})$ satisfying $\operatorname{Re}(\lambda(\mathbf{k}))>0$ (and hence an instability) provided that either $\operatorname{tr}\left(\mathbf{Q}\left(|\mathbf{k}|^{2}, \boldsymbol{\mu}\right)\right)>0$ or $\operatorname{det}\left(\mathbf{Q}\left(|\mathbf{k}|^{2}, \boldsymbol{\mu}\right)\right)<$ 0 . Due to the assumption of stable reaction kinetics at a spatially uniform steady state, we have $\operatorname{tr}(\mathbf{Q}(0, \boldsymbol{\mu}))=-\left(a_{11} u_{1}^{*}+a_{22} u_{2}^{*}\right)<0$ and $\operatorname{det}(\mathbf{Q}(0, \boldsymbol{\mu}))=\left(a_{11} a_{22}-a_{12} a_{21}\right) u_{1}^{*} u_{2}^{*}>0$. Furthermore, from the form of $(43)$, we have $\operatorname{tr}\left(\mathbf{Q}\left(|\mathbf{k}|^{2}, \boldsymbol{\mu}\right)\right)<\operatorname{tr}(\mathbf{Q}(0, \boldsymbol{\mu}))<0$ for all $|\mathbf{k}|>0$. Therefore only the condition on the determinant can lead to an instability. When $\mathbf{k}=\mathbf{0}$ we have $\operatorname{det}(\mathbf{Q}(0, \boldsymbol{\mu}))>0$, yet $\operatorname{det}(\mathbf{Q}(0, \boldsymbol{\mu})) \sim \delta_{1} \delta_{2}|\mathbf{k}|^{4} \rightarrow \infty$ in the limit $|\mathbf{k}| \rightarrow \infty$. As such, unstable wave numbers are confined to a bounded region $0<K_{1} \leq|\mathbf{k}| \leq K_{2}<\infty$, if they exist at all.

Using the expression in (44), we obtain parameter sets leading to instability from requiring that the condition $\operatorname{det}\left(\mathbf{Q}\left(|\mathbf{k}|^{2}, \boldsymbol{\mu}\right)\right)<0$ hold for at least some $\mathbf{k}$. We then solve (6) using the finite-element approach as before. In all cases, we focus on varying parameters $b_{12}$ and $b_{21}$. While in the fecundity $E_{\ell}$ these parameters are both positive for competition dynamics (denoting repulsive interactions) in reality one species might move toward another in the event that the second species has located a resource or avoids a hazard, or for mutual protection through strength in numbers. In the predator-prey or generalist predator-prey dynamics, a predator population might actually move away from prey if already satiated, or if the presence of a prey might denote a super predator (as in the case of some tritrophic plant interactions (Heil, 2008)). Conversely, a prey population might risk moving toward predators if the predators are able to locate scarce resources such as water. We note that such mismatches between actual and perceived risks are common, and have been implicated in a variety of species and interactions (Laundré et al, 2010; Madin et al, 2011), suggesting that the original model of movement along fecundity gradients is at best an idealization.

We first consider competition dynamics with increased inter-species repulsion (compared to movement along fecundity gradients) in Figure 4. In Figure $4(\mathrm{a})$ we show values of $b_{12}$ and $b_{21}$ such that the homogeneous equilibrium is only just past the instability threshold, yet we observe a large separation of the two populations into distinct colonies. If we increase $b_{21}$, we see that the second species recedes into a small portion of the domain, leaving the other population free to inhabit most of the domain (Figure 4(b)). If instead both of these advection parameters are set well above the corresponding interspecies fecundity, then the populations separate completely, leaving a barren region in between their separate colonies. Such completely-disconnected colonies are not typical of reaction-diffusion models, but are reminiscent of populations avoiding one another over large regions (Madin et al, 2011).

The expression in (44) has immediate consequences for predator-prey interactions. Without loss of generality, assume that $u_{2}$ preys on $u_{1}$, so that $a_{21}<0$ and $a_{21}>0$. We then see that if the advection parameters are either zero, or have the same sign as fecundity, then no term in (44) is negative, preventing any instability of this kind from occurring. We note that the self-advection parameters $b_{11}$ and $b_{22}$ can lead to numerical difficulties, and in some cases blow-up due to population aggregation in increasinglyfine regions (which also breaks some of the assumptions needed to justify the use of a spatially continuous domain). Therefore we leave these as positive or zero, and consider changing the signs of the interaction parameters in the next two examples.

If $b_{21}>0$, this suggests a predator which moves away from prey, possibly due to visual markings or other cues which make the predator avoid the prey. In all such parameters we explored, we observed spatiotemporal behaviors, rather than stationary patterns. In Figure 5 we show simulations in such a case which lead to spatiotemporal evolution of the predator and prey densities. The prey stay close to their predator-free carrying capacity in most of the domain except for some pockets along the boundaries 
(a)

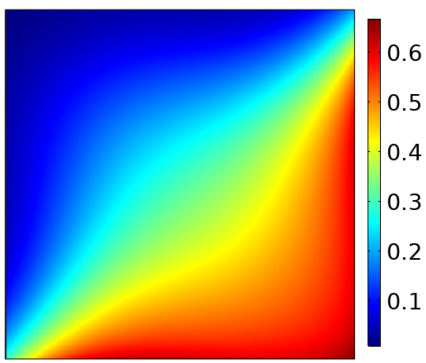

(b)

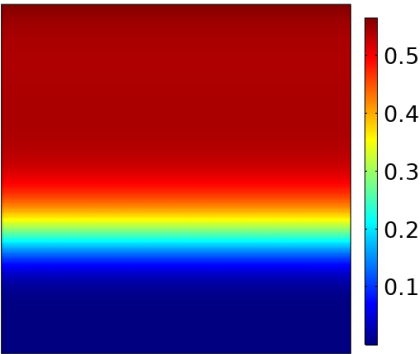

(c)

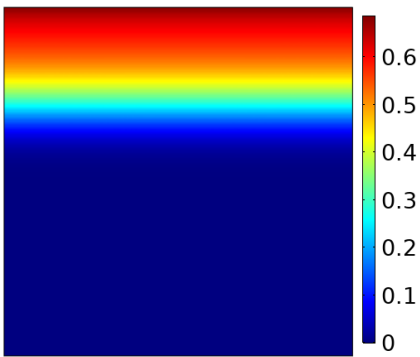

$u_{1}$
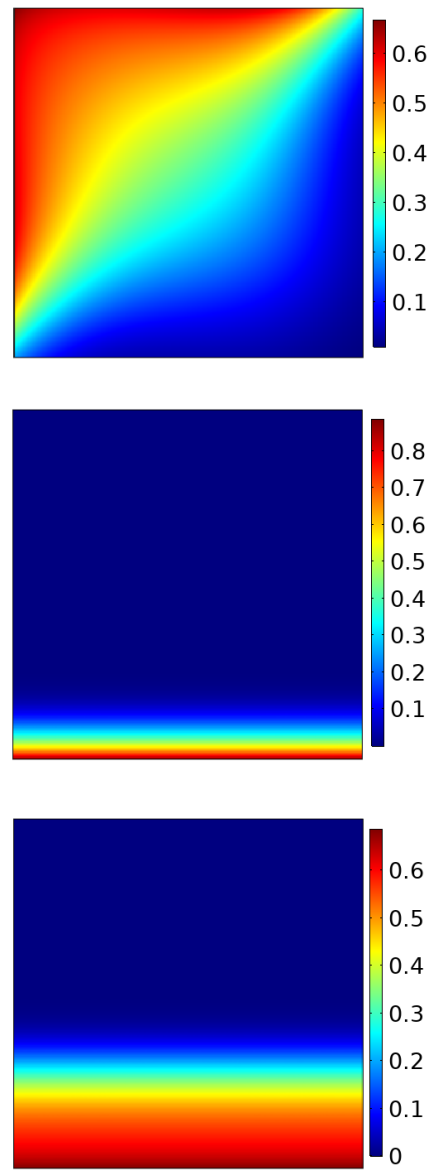

$u_{2}$

Fig. 4 Simulations of (6) showing competition dynamics with $\delta_{1}=\delta_{2}=0.01, \mu_{1}=\mu_{2}=0.1, \epsilon_{j}=0.1, a_{11}=a_{22}=2$, $a_{12}=a_{21}=1, A_{1}=A_{2}=1, b_{11}=b_{22}=2$, with (a) $b_{12}=3, b_{21}=3$, (b) $b_{12}=3, b_{21}=10$, (c) $b_{12}=b_{21}=10$. These are recorded at time $t=200$, though the densities have been approximately at a steady state since $t=20$.

and the interior where colonies of predator exist. The isolated predator colony in the top-right of the domain, as well as those along the boundaries, remain in approximately the same position for $t=200$ seconds, suggesting a balance between diffusion and advection which leads to a coexistence in a patterned state. The other interior colonies emerge spontaneously from regions of low predator density, and drift toward one another due to the larger advective force from the surrounding prey, merging into a colony which decays in size over time, as they have no source of sustenance within larger colonies. While it is unlikely for a canonical predator-prey system to exhibit the kind of interactions shown here, it leads to a non-intuitive niche formation, as the predators gain no advantage from being in larger colonies, yet pursue this due to the external pressure from the prey. We note that allowing the predator to make use of a resource (i.e. setting $A_{2}>0$ ), we observe larger colonies with similar behaviors to what is shown in this example, as the predator colonies can become somewhat self-sustaining with this additional resource.

Finally we consider the case of $b_{12}<0$, corresponding to prey being attracted to predators. This is a more typical interaction than predators running from prey, as there are classes of ambush predators across a variety of different taxa which lure prey using different means (for instance, adders have been reported to employ several means to attract prey (Hagman et al, 2008)). Here we only consider those which interactions where prey move directly up predator gradients, but note that the formalisms developed by Potts and Lewis (2019) could apply to a variety of such luring tactics. In all of the parameters we explored satisfying (44), this interaction led to spatiotemporal behavior. We give an example in Figure 6, where we see very long-time oscillations between stripe configurations of both predator and prey density. After an initial transient pattern of movement, the populations appear to oscillate between a horizontal and a vertical stripe, with a transition through a square-like configuration, with the predator seemingly more localized than the prey. These oscillations persist for at least $t=10000$ units of time, suggesting that this behavior may be stable. We remark that neither predator nor prey ever reaches a low density 

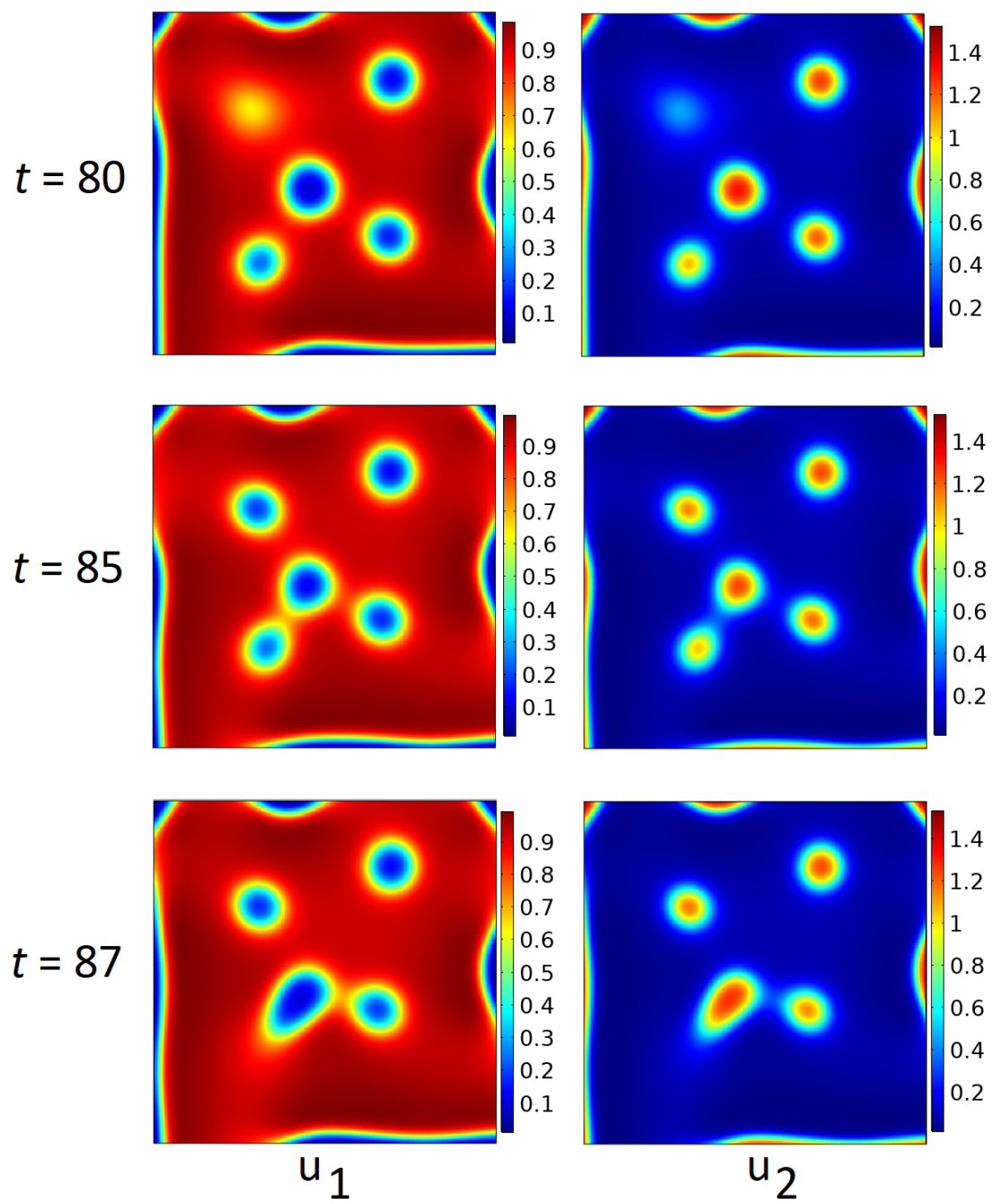

Fig. 5 Simulations of (6) showing predator $\left(u_{2}\right)$ - prey $\left(u_{1}\right)$ dynamics with $\delta_{1}=\delta_{2}=0.01, \mu_{1}=\mu_{2}=0.1, \epsilon_{j}=0.001$, $a_{11}=a_{22}=1, a_{12}=1, a_{21}=-1, A_{1}=1, A_{2}=0, b_{11}=b_{22}=b_{12}=1, b_{21}=2$

in any part of the domain, and so the oscillations concern the densest regions of each population, but that the species coexist throughout the entire domain.

\section{Interplay between Turing patterning and spatially heterogeneous resources}

There have been a variety of studies on the emergence or suppression of Turing structures in spatially heterogeneous models or domains (Benson et al, 1993; Cobbold et al, 2015; Krause et al, 2018b; Page et al, 2003, 2005). Kurowski et al (2017) showed that for two species, colony formation was possible due to heterogeneous spatial forcing, such as that due to a spatially non-uniformly distributed resource or hazard (and segregation effects have also been observed in simple models of competitors in heterogeneous domains (Ali and Cosner, 1995)). However, for two species in a homogeneous domain, the Turing mechanism is not possible for spatial patterning, as shown in Section 3.2, and in Kurowski et al (2017) under some restrictions. In the present section, we consider parameter regimes for which there is a diffusion driven instability leading to pattern formation, and generalize these dynamics to the case of heterogeneous spatial resources or hazards, to better understand the role that the interplay between these two features will play in colony formation.

We generalize fecundity functions $E_{\ell}$ in $(6 \mathrm{c})$ to the form

$$
E_{\ell}\left(u_{1}, u_{2}, \ldots, u_{n}, \mathbf{x}\right)=A_{\ell}(\mathbf{x})-\sum_{j=1}^{n} a_{\ell j} u_{j}
$$




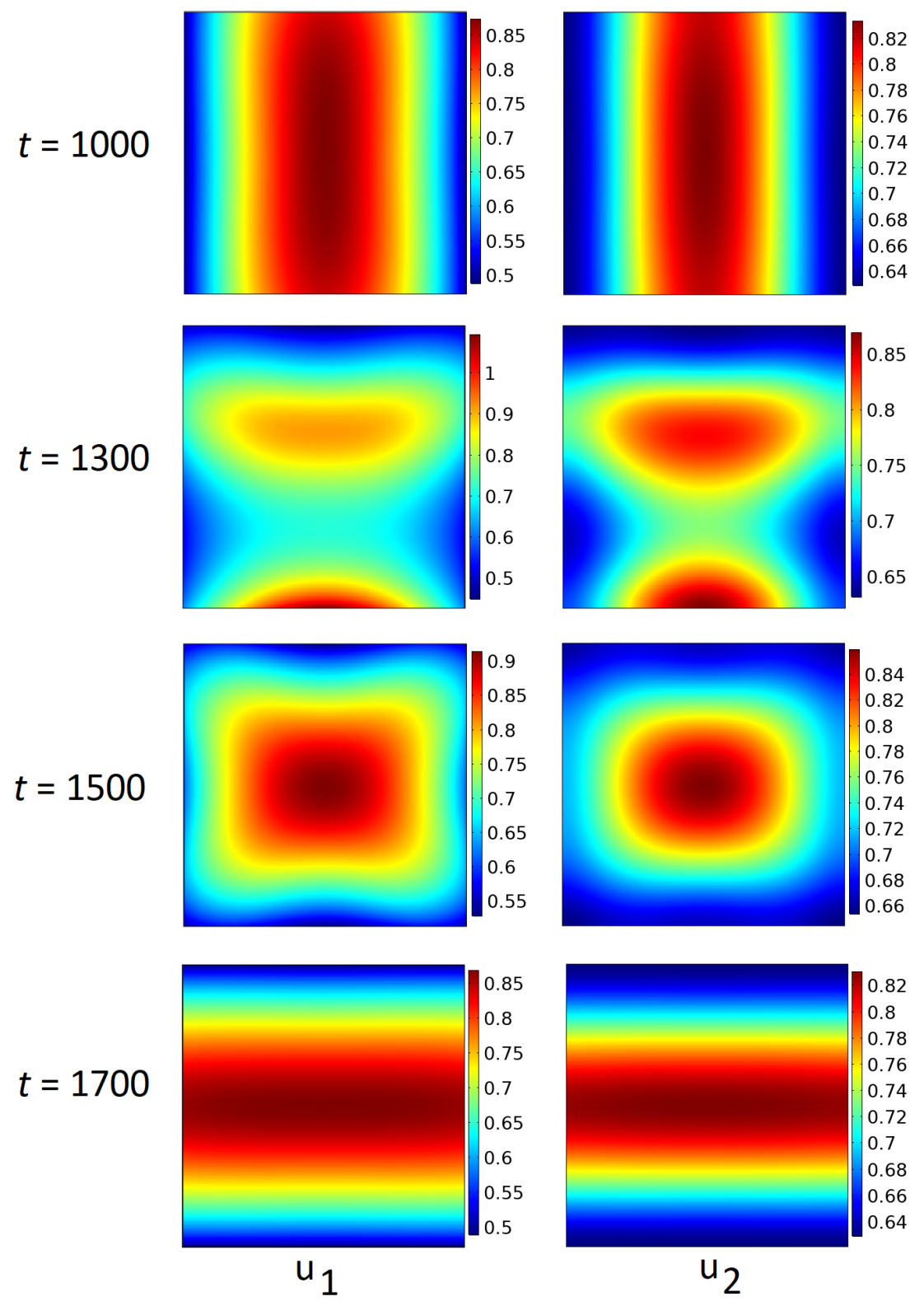

Fig. 6 Simulations of (6) showing predator $\left(u_{2}\right)$ - prey $\left(u_{1}\right)$ dynamics with $\delta_{1}=\delta_{2}=0.01, \mu_{1}=\mu_{2}=0.1, \epsilon_{j}=0.001$, $a_{11}=a_{22}=1, a_{12}=1, a_{21}=-1, A_{1}=1.5, A_{2}=0, b_{11}=b_{22}=b_{21}=0, b_{12}=-1$

so that we may have spatial variation in the resource (or, hazard) distribution across the domain. If $A_{\ell}$ is constant, results of the previous sections hold. We shall furthermore consider only $F_{\ell}$ satisfying $\nabla F_{\ell}=\nabla E_{\ell}$, since we focus on diffusive instabilities.

We modify the spatial perturbation (11) to the form

$$
\begin{aligned}
\mathbf{u}(\mathbf{x}, t) & =\mathbf{u}^{*}(\mathbf{x})+\sigma \hat{\mathbf{u}}(\mathbf{x}, t), \\
\mathbf{w}_{\ell}(\mathbf{x}, t) & =\mathbf{w}_{\ell}^{*}(\mathbf{x})+\sigma \hat{\mathbf{w}}_{\ell}(\mathbf{x}, t),
\end{aligned}
$$

Placing (46) into (6), and expanding in the small parameter $\sigma$, at leading order $\mathcal{O}(1)$ we have

$$
\begin{gathered}
\delta_{\ell} \nabla^{2} u_{\ell}^{*}-\mu_{\ell} \nabla \cdot\left(u_{\ell}^{*} \mathbf{w}_{\ell}^{*}\right)+u_{\ell}^{*} E_{\ell}\left(\mathbf{u}^{*}, \mathbf{x}\right)=0, \\
-\epsilon_{i} \nabla^{2} \mathbf{w}_{\ell}^{*}+\mathbf{w}_{\ell}^{*}=\nabla E_{\ell}\left(\mathbf{u}^{*}, \mathbf{x}\right)=0,
\end{gathered}
$$

which govern the structure of the steady state solutions.

Note that (47) is a nonlinear PDE system with spatially heterogeneous terms, the systematic study of which is beyond the scope of the present paper. In order to make further analytical progress, we assume $A_{\ell}(\mathbf{x})=A_{0, \ell}+\sigma A_{1, \ell}(\mathbf{x})$, where $|\sigma| \ll 1$ is a small parameter. For this case, $A_{\ell}(\mathbf{x})$ is constant at leading 
order, with the spatial variation being small by comparison to the base state. Under such an assumption, we can adapt the analysis of Section 3 to study this case.

At lowest order, we have the spatially uniform solution $\mathbf{u}^{*}$ where $u_{\ell}^{*}\left(A_{0, \ell}-\sum_{j=1}^{n} a_{\ell j} u_{j}^{*}\right)=0$, while $\mathbf{w}_{\ell}^{*}=0$, as in Section 3.1. The $O(\sigma)$ perturbation terms used in the stability analysis are then governed by the system

$$
\begin{gathered}
\frac{\partial \hat{u}_{\ell}}{\partial t}=\delta_{\ell} \nabla^{2} \hat{u}_{\ell}-\mu_{\ell} u_{\ell}^{*} \nabla \cdot \hat{\mathbf{w}}_{\ell}+u_{\ell}^{*} A_{1, \ell}(\mathbf{x})+\hat{u}_{\ell}\left(A_{0, \ell}-\sum_{j=1}^{n} a_{\ell j} u_{j}^{*}\right)-u_{\ell}^{*}\left(\sum_{j=1}^{n} a_{\ell j} \hat{u}_{j}\right) \\
-\epsilon_{\ell} \nabla^{2} \hat{\mathbf{w}}_{\ell}+\hat{\mathbf{w}}_{\ell}=\nabla A_{1, \ell}(\mathbf{x})-\sum_{j=1}^{n} a_{\ell j} \nabla \hat{u}_{j} .
\end{gathered}
$$

As the system (48) is linear, then by superposition principle we may write

$$
\begin{aligned}
\hat{\mathbf{u}}(\mathbf{x}, t) & =\hat{\mathbf{u}}_{\text {hom }}(\mathbf{x}, t)+\hat{\mathbf{u}}_{\text {static }}(\mathbf{x}), \\
\hat{\mathbf{w}}_{\ell}(\mathbf{x}, t) & =\hat{\mathbf{w}}_{\ell, \operatorname{hom}}(\mathbf{x}, t)+\hat{\mathbf{w}}_{\ell, \text { static }}(\mathbf{x}),
\end{aligned}
$$

where the functions with the "static" subscript hold the spatial variations due the the spatial forcing function $A_{1, \ell}(\mathbf{x})$ and are governed by the stationary system

$$
\begin{gathered}
\delta_{\ell} \nabla^{2} \hat{u}_{\ell, \text { static }}-\mu_{\ell} u_{\ell}^{*} \nabla \cdot \hat{\mathbf{w}}_{\ell, \text { static }}+u_{\ell}^{*} A_{1, \ell}(\mathbf{x})+\hat{u}_{\ell, \text { static }}\left(A_{0, \ell}-\sum_{j=1}^{n} a_{\ell j} u_{j}^{*}\right)-u_{\ell}^{*} \sum_{j=1}^{n} a_{\ell j} \hat{u}_{j, \text { static }}=0 \\
-\epsilon_{\ell} \nabla^{2} \hat{\mathbf{w}}_{\ell, \text { static }}+\hat{\mathbf{w}}_{\ell, \text { static }}=\nabla A_{1, \ell}(\mathbf{x})-\sum_{j=1}^{n} a_{\ell j} \nabla \hat{u}_{j, \text { static }}
\end{gathered}
$$

while the functions with the "hom" subscript hold the spatiotemporal variations and are governed by the homogeneous yet time dependent system

$$
\begin{gathered}
\frac{\partial \hat{u}_{\ell, \mathrm{hom}}}{\partial t}=\delta_{\ell} \nabla^{2} \hat{u}_{\ell, \mathrm{hom}}-\mu_{\ell} u_{\ell}^{*} \nabla \cdot \hat{\mathbf{w}}_{\ell, \mathrm{hom}}+\hat{u}_{\ell, \mathrm{hom}}\left(A_{0, \ell}-\sum_{j=1}^{n} a_{\ell j} u_{j}^{*}\right)-u_{\ell}^{*}\left(\sum_{j=1}^{n} a_{\ell j} \hat{u}_{j, \mathrm{hom}}\right) \\
-\epsilon_{\ell} \nabla^{2} \hat{\mathbf{w}}_{\ell, \mathrm{hom}}+\hat{\mathbf{w}}_{\ell, \mathrm{hom}}=-\sum_{j=1}^{n} a_{\ell j} \nabla \hat{u}_{j, \mathrm{hom}} .
\end{gathered}
$$

The spatial forcing will therefore not impact the temporal properties of the perturbation (46), with the spatial forcing just being added to the temporal terms in the perturbation. As such, temporal growth leading to diffusive instability will arise only from the "hom" term in the perturbations.

Assuming $\hat{\mathbf{u}}_{\mathrm{hom}}(\mathbf{x}, t)=\tilde{\mathbf{u}} \exp (\lambda t+\mathrm{i} \mathbf{k} \cdot \mathbf{x})$ and $\hat{\mathbf{w}}_{\ell, \text { hom }}(\mathbf{x}, t)=\tilde{\mathbf{w}}_{\ell} \exp (\lambda t+\mathrm{i} \mathbf{k} \cdot \mathbf{x})$ where $\tilde{\mathbf{u}}$ and $\tilde{\mathbf{w}}_{\ell}$ are constant vectors (akin to what was assumed in (11)), the system (51) reduces to the previously derived algebraic system (12). From here, the instability analysis follows exactly as in Section 3.1. Therefore, in the case where spatial variations in the fecundity are small, there is no appreciable difference in the stability analysis compared to that which was outlined for diffusive instabilities studied in Section 3. In this regime, we anticipate a superposition of spatial structure due to Turing patterns and heterogeneous spatial forcing.

In order to numerically study colony formation from the interaction of Turing patterns and spatial heterogeneity, we shall make use of products of functions taking the form

$$
B\left(s ; s_{1}, s_{2}, \beta\right)=\tanh \left(\beta\left(s-s_{1}\right)\right)-\tanh \left(\beta\left(s-s_{2}\right)\right) .
$$

Parameters $s_{1}$ and $s_{2}$ control the position of the additional resource, while $\beta$ controls the how sharp the transition between the resource and the rest of the domain is.

For the plots shown in Figure 7 we make use of resource terms of the form

$$
\begin{aligned}
& A_{1}(\mathbf{x})=16\left(1+0.001 \times B\left(x_{1} ; 0.3,0.7,100\right) B\left(x_{2} ; 0.3,0.7,100\right)\right) \\
& A_{2}(\mathbf{x})=25\left(1+0.001 \times B\left(x_{1} ; 0.2,0.8,100\right) B\left(x_{2} ; 0.2,0.8,100\right)\right) \\
& A_{3}(\mathbf{x})=-9\left(1-0.001 \times B\left(x_{1} ; 0.4,0.6,200\right) B\left(x_{2} ; 0.4,0.6,200\right)\right) .
\end{aligned}
$$



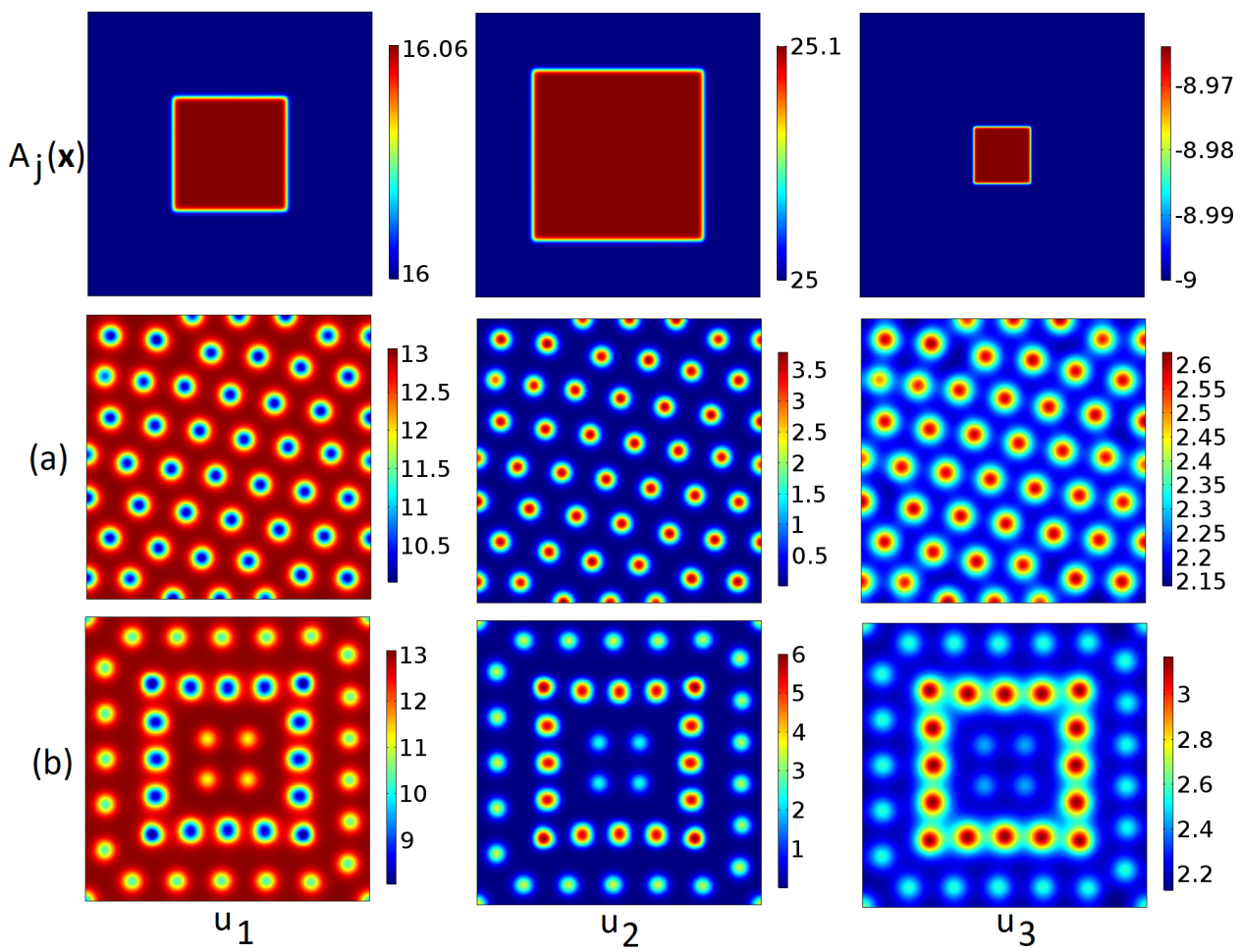

Fig. 7 Simulations of (6) with generalized fecundity (45) showing spatial patterning resulting from generalist predator - prey dynamics according to (38c) in the presence of spatially heterogeneous resources or hazards as described by (53). The values used to produce these plots are given by the reaction kinetic parameters $a_{11}=1.09, a_{12}=0.8, a_{13}=0.81$, $a_{21}=1.64, a_{22}=1.1, a_{23}=1.66, a_{31}=-0.84, a_{32}=-1.7, a_{33}=1.11$, while the diffusion parameters satisfy $\delta_{1}=10^{-4}$, $\delta_{2}=6.25 \times 10^{-6}, \delta_{3}=8.1 \times 10^{-3}$. We ignore advection in this case, by setting $\mu_{\ell}=0, \ell=1,2,3$. In row (a) we take homogeneous resources and hazards given by $A_{1}=16, A_{2}=25, A_{3}=-9$, while in (b) we take spatially heterogeneous resources and hazards according to (53). The simulations are shown at time $t=35000$, by which point the dynamics have converged to steady states.

These variations correspond to regions which are more favorable than their surrounding regions. In Figure 7 we plot these functions. We also plot simulations of (intraguild) predator - prey dynamics with two prey, assuming constant resources in Figure $7(\mathrm{a})$, along with the dynamics under spatially heterogeneous resources (53) in Figure 7(b).

Comparing the two simulations in Figure 7, we find that prey species $u_{1}$ is better off within the small square on which its resources $A_{1}(\mathbf{x})$ have improved, whereas prey species $u_{2}$ is best off in the outer region on which its resources $A_{2}(\mathbf{x})$ have improved, but not inside the overlap with the smaller region on which $A_{1}(\mathbf{x})$ has also improved. The reason for the behavior of $u_{2}$ may be due to competitive exclusion, due to the increased concentration of $u_{1}$ in the smaller central region. To compete successfully, $u_{2}$ positions its greatest regions of concentrations outside that of $u_{1}$, but still within the spatial region on which resources are enhanced. Conversely, on this overlap region we find that $u_{1}$ tends to be worse off, even worse than on the outer region where the resources are not enhanced, due to the fact that on this intermediate region $u_{2}$ has an advantage over $u_{1}$ which it does not have on the outer region. This, again, could be due to competitive exclusion, with $u_{1}$ developing a niche for the innermost region and $u_{2}$ developing a niche for the intermediate region.

The choice of $A_{3}(\mathbf{x})$ in (53) means that the predator is subject to a hazard rather than a resource, with the central region still a hazard but less so than the surrounding region. However, we find in Figure 7 that the predator $u_{3}$ has maximal concentration near the peak concentration of prey $u_{2}$, and the concentration is actually fairly low on the center region. Therefore, for this particular choice of parameters, the predator is better off in a slightly more hazardous region provided there is an abundance of prey. We also note that the predator $u_{3}$ has a preference for prey $u_{2}$ over prey $u_{1}$ (since $\left|a_{13}\right|<\left|a_{23}\right|$ ), and this is reflected in the simulations. 


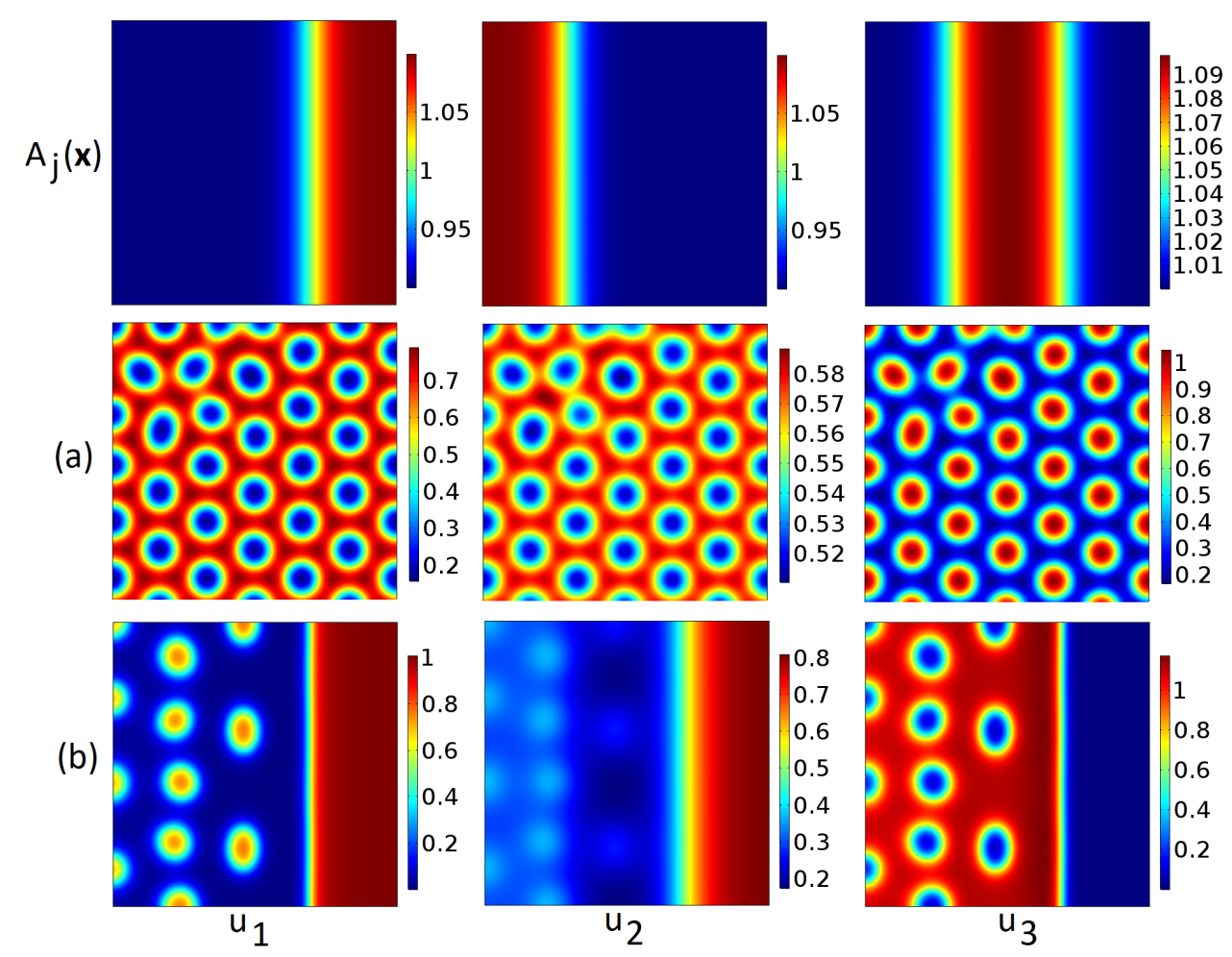

Fig. 8 Simulations of (6) with generalized fecundity (45) showing spatial patterning resulting from generalist predator - prey dynamics according to (38f) in the presence of spatially heterogeneous resources or hazards as described by (54). The values used to produce these plots are given by the reaction kinetic parameters $a_{11}=1.09, a_{12}=0, a_{13}=0.81$, $a_{21}=0, a_{22}=1.1, a_{23}=0.83, a_{31}=1.68, a_{32}=-0.85, a_{33}=1.11$, while the diffusion parameters satisfy $\delta_{1}=10^{-5}$, $\delta_{2}=4 \times 10^{-3}, \delta_{3}=\times 10^{-5}$. In (a) we set $\mu_{\ell}=0, \ell=1,2,3$, so that there is no advection, while in (b) we set $\mu_{1}=10^{-3}$, $\mu_{2}=10^{-4}, \mu_{3}=\times 10^{-5}$. In row (a) we take homogeneous resources given by $A_{\ell}=1, \ell=1,2,3$, while in (b) we take spatially heterogeneous resources and hazards according to (54). The simulations are shown at time $t=35000$, by which point the dynamics have converged to steady states.

For the plots shown in Figure 8 we make use of resource terms of the form

$$
\begin{aligned}
& A_{1}(\mathbf{x})=1+0.1 \times \tanh (12(x-0.7)), \\
& A_{2}(\mathbf{x})=1-0.1 \times \tanh (12(x-0.3)), \\
& A_{3}(\mathbf{x})=1-0.05 \times \tanh (12(x-0.7))+0.05 \times \tanh (12(x-0.3)) .
\end{aligned}
$$

This corresponds to a domain with split regions favoring different species.

In Figure 8(a) we provide simulations with spatially uniform resources, in order to demonstrate the emergence of static Turing patterns. In Figure 8(b) we then utilize the spatially heterogeneous resources defined in (54). The generalist predator $u_{3}$ does quite well in the central region of the domain, even though the improvement in the resource was quite small. The prey species $u_{2}$ is suppressed in this middle region, both due to poorer resources and greater predation. Meanwhile, the generalist predator $u_{3}$ does poorly on the right-most region of the domain, which frees up the region for colonization by prey species $u_{2}$. Note that the species $u_{1}$ is not prey for $u_{3}$ nor a predator of $u_{2}$, rather it avoids and is avoided by the predator $u_{3}$, and this can be seen in the disjoint regions as each of $u_{1}$ and $u_{3}$ are found in simulations shown in both (a) and (b). These dynamics can therefore be seen as a mix of predator-prey and competitive exclusion, with spatial partitioning enhanced by the heterogeneous resources which break the symmetry inherent in pure Turing patterns.

In Figure 9, we explore the effect of heterogeneity when there is an instability corresponding to the case where the condition (33c) changes sign. We consider

$$
\begin{aligned}
& A_{1}(\mathbf{x})=5-5 \times B(x ; 0.35,0.55,50) B(y ; 0.35,0.55,50), \\
& A_{2}(\mathbf{x})=9-5 \times B(x ; 0.35,0.55,50) B(y ; 0.35,0.55,50), \\
& A_{3}(\mathbf{x})=-7-5 \times B(x ; 0.35,0.65,50) B(y ; 0.35,0.65,50) .
\end{aligned}
$$

This could represent a lake in which the species will drown, a mountain range or desert which is dangerous to traverse, or some other hazard. We also choose parameter values identical to those of Figure 2. 


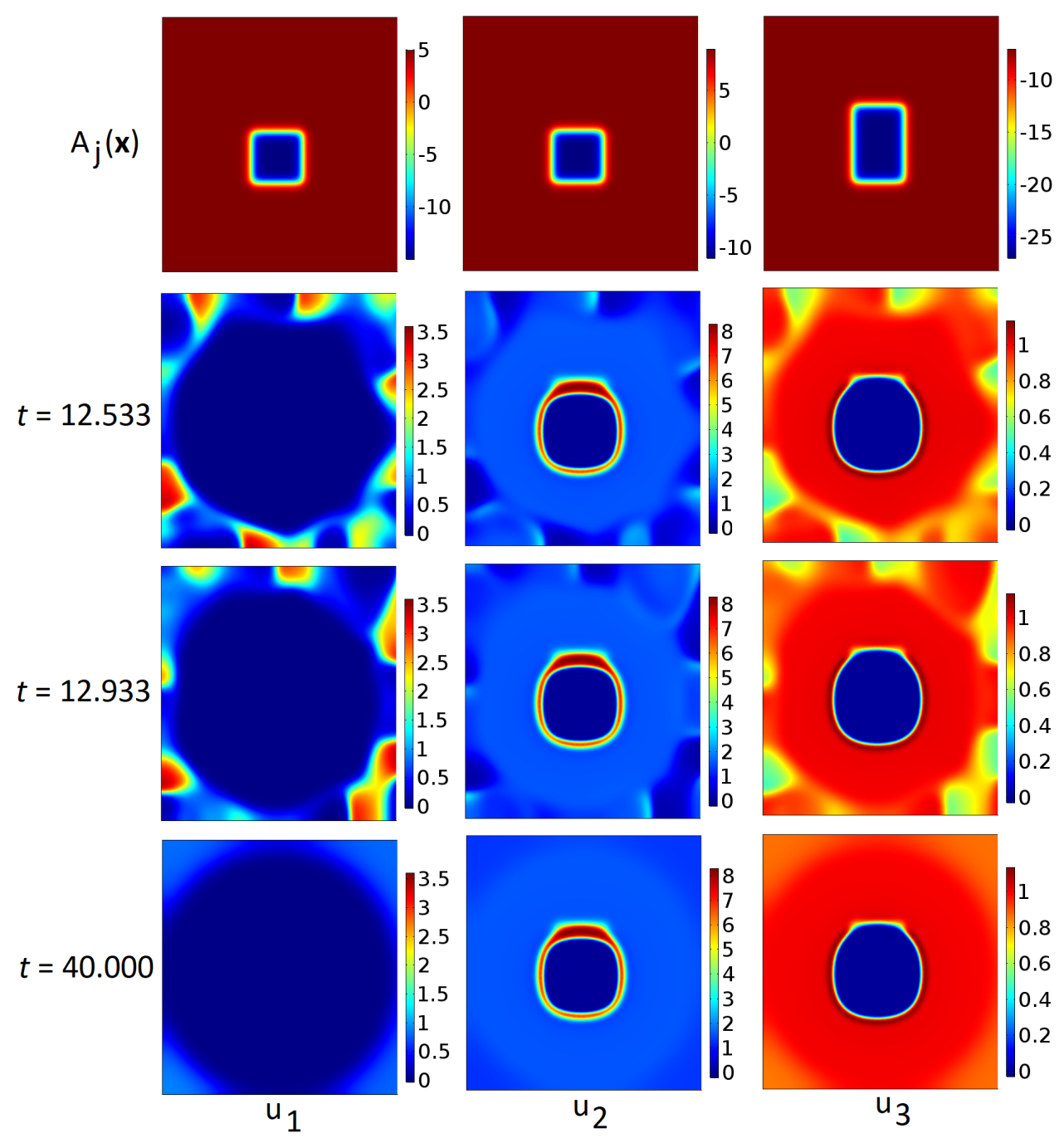

Fig. 9 Simulations of (6) with generalized fecundity (45) showing spatial patterning resulting from generalist predator prey dynamics according to (38f) in the presence of spatially heterogeneous resources or hazards as described by (55). All parameter values are the same as in Figure 2, but with the $A_{\ell}$ 's replaced by the heterogeneous functions defined in (55). We observe interesting initial transient behavior; the prey species appear to move around the obstacle in a rotational manner. The heterogeneity eventually forces the system into the steady state shown when $t=40$. Note that this is a coexistence state since all of the species survive. All species have zero population in the death zone.

We observe interesting initial transient behavior; the prey species appear to move around the obstacle in a rotational manner. As such, the spatiotemporal nature of the dynamics observed in Figure 2 is preserved here. However, for larger time, this spatial heterogeneity eventually forces the system into a steady state. Unlike for the other cases shown in Figures 7-8, there is a change in the nature of the stability, as these dynamics tend to a steady state whereas the dynamics seen in Figure 2 remain spatiotemporal in nature. This is because the spatial variation in the heterogeneity is quite large, unlike for the cases shown in Figures 7-8 in which it was small. For large spatial variations in the resource or hazard, there is the possibility for more fundamental deviations between predictions from linear stability analysis and the simulations.

Note that the hazard zone described by (55) is larger for the predator species. This results in a small ring of prey species $u_{2}$ persisting just outside the $A_{2}$ hazard zone, but within the $A_{3}$ hazard zone for the predator. On the other hand, the prey species $u_{1}$ is forced toward the outer boundary of the domain, with the predator $u_{3}$ thriving in an annular region in between these two populations. This provides a nice example of spatial partitioning due to heterogeneous placement of resources and hazards. 


\section{Discussion}

We have extended the model previously studied in Grindrod (1988) and Kurowski et al (2017) to account for many species and directed toward gradients which need not be equivalent to fecundity gradients. We then studied a variety of spatial pattern and wave solutions which give rise to aggregation and colony formation. The model (6) employs a non-local cross-diffusion mechanism for advection toward resource gradients, and hence our study focused on the role of this type of advection in modifying solutions which lead to the development and persistence of colony formation. Such a model can be valuable for grounding experimental and verbal models in spatial ecology capturing directed movement within a theoretical framework. We refer to the discussion in Section 1 for other frameworks of modelling directed motion of populations.

In the case of three or more species with directed motion toward their respective fecundity gradients, $\nabla F_{\ell}=\nabla E_{\ell}$, we find that diffusive instabilities and pattern formation may result through the Turing mechanism. We first obtain sufficient analytic criteria for a Turing instability, and then use this to motivate parameter regimes for which we carry out numerical simulations. The Turing instability leads to the development of colonies, which may take a variety of forms depending upon the model parameters. For some parameter values, stationary patterns emerge, corresponding to aggregation leading to static colonies. Here we see highly localized structures, with mutual exclusion for reaction kinetics under which pairs of species mutually repel one another. This is seen in Figures 1 and 3, where we demonstrate various scenarios where colonies form and persist over time. For other parameter values, spatiotemporal structures develop, leading to colonies which move in time, as seen, for instance, in Figure 2. Such spatiotemporal structures are particularly relevant in cases where there are predator-prey dynamics, with predator colonies able to pursue prey colonies. We classified the kinds of community structures which may lead to such instabilities, as shown in (37)-(39), in order to determine which kinds of interspecies interactions lead to such patterning instabilities.

Diffusive instabilities due to the Turing mechanism occur in the model only when three or more species are present when the directed motion of each species is toward their respective fecundity gradient. When only two species are present, the Turing instability does not occur with this manner of directed motion, as shown in Section 3.2, and colony formation through the Turing mechanism does not occur. However, if the directed motion is not along fecundity gradients prescribed by the reaction kinetics, e.g. $\nabla F_{\ell} \neq \nabla E_{\ell}$, then an instability can develop even for the two-species variant of the model (6), leading either to segregated populations or spatiotemporal colony formation. To better motivate the choice $\nabla F_{\ell} \neq \nabla E_{\ell}$, note that advection toward resource gradients as calculated from fecundity $\left(\nabla F_{\ell}=\nabla E_{\ell}\right)$ would be optimal in the sense of population fitness. However, in practice, many species may not be able to actually forage or migrate in an optimal manner. Indeed, over long distances, the species will not have complete information on resources or hazards, and may not be fully aware of other species (either competitors or predators). Due to this lack of information, it is more likely that directed motion of the manner we consider is often sub-optimal in many ecosystems, with populations using their best guess in order to motivate motion in a certain direction, which may lead to less than optimal (but, perhaps still favorable) foraging and migration strategies.

When $\nabla F_{\ell} \neq \nabla E_{\ell}$, we considered the case of two species, as this is the lowest number of species for which an instability manifests. In comparison with the diffusive instabilities, we find that these advection induced instabilities result in pattern formation which is dominated more by advection, and the resulting patterns are global over the spatial domain, rather than strongly localized in space as in the standard Turing case; see, for instance, Figure 4. The reason for this likely lies in the non-local manner of advection, with species directed to move greater distances to form colonies, whereas colony formation is far more localized when diffusion dominates. Such instabilities were only possible for predator-prey interactions under unusual scenarios (predators fleeing from prey or prey seeking out predators), and these led to interesting spatiotemporal movements which were more structured than the more chaotic movements shown in Figure 2. We anticipate that more complex spatiotemporal patterns may occur under more general functional responses, particularly those incorporating Allee effects which allow for group defense or other mechanisms by which these interactions can arise.

Heterogeneous spatial forcing through the assumption that $A_{\ell}$ is a function of the spatial coordinates results in spatial pattern formation, even if the equations do not admit patterns in the case of homogeneous equations, as was previously shown in Kurowski et al (2017). However, Kurowski et al (2017) considered a two-species model incapable of exhibiting pattern forming instabilities in the homogeneous setting. When there are three or more species, the model (6) allows for Turing instability-induced patterning, and so it is natural to consider how these two mechanisms will interact to allow aggregation of 
populations leading to colony formation. Taking parameter regimes corresponding to diffusive instabilities studied in Section 3, considered various resource and hazard distributions. We were able to show analytically and through simulations that when the spatial heterogeneity of the resources $\left(A_{\ell}>0\right)$ or hazards $\left(A_{\ell}<0\right)$ was small, the emergent solutions had properties of both Turing patterns and patterns resulting from spatial forcing due to $A_{\ell}$. This is best seen in Figures 7-8, where we compare Turing patterns in row (a) with patterns resulting from a combination of the Turing instability and spatially heterogeneous forcing due to $A_{\ell}$. While species still form colonies, these colonies are no longer uniformly distributed throughout the domain; instead the colonies are more likely in regions which are more favorable to a given species. Note, however, that when an environment is more favorable to two or more species, not all species will be better off. Rather, the species with the most favorable fecundity will tend to do best to the possible detriment of its competitors, and therefore enrichment of an environment may actually result in one species doing worse. Conversely, hazards may result in some species being particularly worse off locally (if they are most sensitive to the hazard), which gives other species the opportunity to do better than they would have without the hazard. We also find that the presence of large enough spatial forcing can suppress spatiotemporal patterning, leading to stationary spatial patterns and hence greater stability of certain ecosystems; compare, for instance, Figure 2 with Figure 9.

We note that in many of the three-population interactions, one of the populations remains more homogeneous than the other, exhibiting Turing-like spatial oscillations around an equilibrium concentration. The other two species in these cases are locally driven between a near-extinction state and a two-species equilibrium state, suggesting patterning due to multistability mediated by the species with small spatial variations (see Figures 1, 3, 7-8). This is also apparent in the spatiotemporal behavior shown in Figure 2. In contrast, two of the examples of the advection-dominated two-species patterns exhibit a much stronger segregation effect mediated between local equilibria (see Figures 4-5). This further suggests that the patterning mechanism is quite different in this case, though with similar local niches forming as in two populations in the three-species examples.

Our findings suggest the emergence of niche partitioning, according to local environmental cues, in the spatially forced models. Although Turing patterns may develop on homogeneous domains, such patterns are often uniformly and randomly distributed throughout the domain. With the resource (or hazard) distribution given by $A_{\ell}$ taken as a function of space, colony formation in each species is either enhanced or suppressed locally. Niche partitioning in a spatial context has been observed in various ecosystems (Albrecht and Gotelli, 2001; Fitzsimons et al, 2008; Lorenzetti et al, 1997; Lyson and Longrich, 2010; Quillfeldt et al, 2013; Schuette et al, 2013; Winder, 2009), making the model (6) potentially attractive for modelling a variety of specific ecosystems. Krause et al (2020) shows that for heterogeneous reactiondiffusion systems, Turing-type instabilities will localize on regions that parametrically satisfy the criteria one would get from a linear stability analysis (under the assumption that the heterogeneity varies slowly compared to any possible emergent patterning). We anticipate that such a localization will also be true for these reaction-advection-diffusion systems, allowing a clearer decoupling between niches which exist due to Turing-type patterns and those which are due to spatial heterogeneity.

While our aforementioned findings are interesting, there are many valuable extensions of the model (6) which could prove useful for other applications. We have taken Lotka-Volterra reaction kinetics for the inter- and intra-species interactions, as these have been used previously for these kinds of models (Grindrod, 1988, 1991; Kurowski et al, 2017). However, there are a variety of other kinetics which may be useful in the study of spatially extended competition or predator-prey models (Fasani and Rinaldi, 2011; Garvie and Golinski, 2010). It is known that Lotka-Volterra models of two interacting species with simple diffusion do not support pattern formation in homogeneous space, and there are multiple ways that such models can be extended to produce pattern formation. As we have shown, one way is to keep the two species Lotka-Volterra structure but replace the simple diffusion with advective movement along gradients of something other than fitness, as we demonstrated in Section 4. When more than two species are present, the Lotka-Volterra structure permits pattern formation through the Turing mechanism, as shown in Section 2 (upon setting the advection terms to zero). Another way to obtain pattern formation in two species predator-prey models under simple diffusion is to generalize the Lotka-Volterra structure to allow either an Allee effect in the prey (Petrovskii et al, 2002; Morozov et al, 2006) or a functional response with mutual interference by the predators, for example a Beddington-DeAngelis functional response Alonso et al (2002). More generally, the problem of when a certain functional response allows for the Turing instability under simple diffusion in predator-prey systems was discussed by (Fasani and Rinaldi, 2011). While our paper shows that a two species Lotka-Volterra structure along with advective movement allows for the formation of spatial patterns, future work will consider the classification of general two species functional response terms which permit pattern formation in conjunction with advective motion. 
Besides directed motion which is along linear gradients in population density, there are a variety of other ways in which animals may choose to move (Potts and Lewis, 2019). Regarding spatially heterogeneous resources, it is possible to also consider seasonally varying or general time-dependent resources, and this was done in Kurowski et al (2017). However, another possibility is to treat the resources with their own governing equations, as the input and decay of these resources over time might be better modelled via dynamical interactions. This was done in recent studies of spatially extended predator - prey systems with allochthonous resources (Bassett et al, 2017; Jansen and Van Gorder, 2018), and extending the $A_{\ell}$ in a similar manner is certainly feasible under the general framework of (6). This extension would allow for the resource to be input or grow at a certain rate, and also to be depleted at a certain rate by the species exploiting it, or by various exogenous effects. The inclusion of such resources may improve predictions of the model (6) for certain ecosystems. Of course the most biologically valuable extensions would involve more ecologically-realistic interactions and dispersal mechanisms, such as accounting for Allee effects and other functional responses.

\section{References}

Abrams PA (2007) Habitat choice in predator-prey systems: spatial instability due to interacting adaptive movements. The American Naturalist 169(5):581-594

Abrams PA, Cressman R, Křivan V (2007) The role of behavioral dynamics in determining the patch distributions of interacting species. The American Naturalist 169(4):505-518

Ackland GJ, Gallagher ID (2004) Stabilization of large generalized Lotka-Volterra foodwebs by evolutionary feedback. Physical review letters 93(15):158701

Albrecht M, Gotelli N (2001) Spatial and temporal niche partitioning in grassland ants. Oecologia 126(1):134-141

Ali SW, Cosner C (1995). Models for the effects of individual size and spatial scale on competition between species in heterogeneous environments. Mathematical biosciences 127(1):45-76

Allen AM, Singh NJ (2016) Linking movement ecology with wildlife management and conservation. Frontiers in Ecology and Evolution 3:155

Alonso D, Bartumeus F, Catalan J (2002) Mutual interference between predators can give rise to Turing spatial patterns. Ecology 83(1):28-34

Amarasekare P (2007) Spatial dynamics of communities with intraguild predation: the role of dispersal strategies. The American Naturalist 170(6):819-831

Andresén P, Bache M, Mosekilde E, Dewel G, Borckmanns P (1999) Stationary space-periodic structures with equal diffusion coefficients. Physical Review E 60(1):297

Armsworth PR, Roughgarden JE (2005a) Disturbance induces the contrasting evolution of reinforcement and dispersiveness is directed and random movers. Evolution 59(10):2083-2096

Armsworth PR, Roughgarden JE (2005b) The impact of directed versus random movement on population dynamics and biodiversity patterns. The American Naturalist 165(4):449-465

Armsworth PR, Roughgarden JE (2008) The structure of clines with fitness-dependent dispersal. The American Naturalist 172(5):648-657

Bassett A, Krause AL, Van Gorder RA (2017) Continuous dispersal in a model of predator-prey-subsidy population dynamics. Ecological Modelling 354:115-122

Baurmann M, Gross T, Feudel U (2007) Instabilities in spatially extended predator-prey systems: Spatiotemporal patterns in the neighborhood of Turing-Hopf bifurcations. Journal of Theoretical Biology 245(2):220-229

Bennett JJ, Sherratt JA (2019) How do dispersal rates affect the transition from periodic to irregular spatio-temporal oscillations in invasive predator-prey systems? Applied Mathematics Letters 94:80-86

Benson DL, Sherratt JA, Maini PK (1993) Diffusion driven instability in an inhomogeneous domain. Bulletin of Mathematical Biology 55(2):365-384

Bernasconi G, Boissonade J (1997) Phyllotactic order induced by symmetry breaking in advected turing patterns. Physics Letters A 232(3-4):224-230

Bertsch M, Gurtin ME, Hilhorst D, Peletier L (1985) On interacting populations that disperse to avoid crowding: preservation of segregation. Journal of Mathematical Biology 23(1):1-13

Bolnick DI, Otto SP (2013) The magnitude of local adaptation under genotype-dependent dispersal. Ecology and Evolution 3(14):4722-4735

Burger M, Di Francesco M, Pietschmann JF, Schlake B (2010) Nonlinear cross-diffusion with size exclusion. SIAM Journal on Mathematical Analysis 42(6):2842-2871 
Cantrell RS, Cosner C, Lou Y, Xie C (2013) Random dispersal versus fitness-dependent dispersal. Journal of Differential Equations 254(7):2905-2941

Chen W, Peng R (2004) Stationary patterns created by cross-diffusion for the competitor-competitormutualist model. Journal of Mathematical Analysis and Applications 291(2):550-564

Chen X, Hambrock R, Lou Y (2008) Evolution of conditional dispersal: a reaction-diffusion-advection model. Journal of Mathematical Biology 57(3):361-386

Cobbold CA, Lutscher F, Sherratt JA (2015) Diffusion-driven instabilities and emerging spatial patterns in patchy landscapes. Ecological complexity 24:69-81

Dawson SP, Lawniczak A, Kapral R (1994) Interaction of Turing and flow-induced chemical instabilities. The Journal of Chemical Physics 100(7):5211-5218

Dillon R, Maini P, Othmer H (1994) Pattern formation in generalized Turing systems. Journal of Mathematical Biology 32(4):345-393

Dubey B, Das B, Hussain J (2001) A predator-prey interaction model with self and cross-diffusion. Ecological Modelling 141(1-3):67-76

Fasani S, Rinaldi S (2011) Factors promoting or inhibiting Turing instability in spatially extended preypredator systems. Ecological Modelling 222(18):3449-3452

Ferreira JD, da Silva SH, Rao VSH (2019) Stability analysis of predator-prey models involving crossdiffusion. Physica D: Nonlinear Phenomena In Press

Fitzsimons MS, Miller RM, Jastrow JD (2008) Scale-dependent niche axes of arbuscular mycorrhizal fungi. Oecologia 158(1):117-127

Fontbona J, Méléard S (2015) Non local Lotka-Volterra system with cross-diffusion in an heterogeneous medium. Journal of Mathematical Biology 70(4):829-854

Galiano G (2011) Modeling spatial adaptation of populations by a time non-local convection crossdiffusion evolution problem. Applied Mathematics and Computation 218(8):4587-4594

Gambino G, Lombardo M, Sammartino M (2008) Cross-diffusion driven instability for a Lotka-Volterra competitive reaction-diffusion system. In: Waves And Stability In Continuous Media, World Scientific, pp 297-302

Gambino G, Lombardo MC, Sammartino M (2012) Turing instability and traveling fronts for a nonlinear reaction-diffusion system with cross-diffusion. Mathematics and Computers in Simulation 82(6):11121132

Gambino G, Lombardo M, Sammartino M (2013) Pattern formation driven by cross-diffusion in a 2d domain. Nonlinear Analysis: Real World Applications 14(3):1755-1779

Garvie MR, Golinski M (2010) Metapopulation dynamics for spatially extended predator-prey interactions. Ecological Complexity 7(1):55-59

Grindrod P (1988) Models of individual aggregation or clustering in single and multi-species communities. Journal of Mathematical Biology 26(6):651-660

Grindrod P (1991) Patterns and waves: The theory and applications of reaction-diffusion equations. Oxford University Press, USA

Gurtin ME, MacCamy RC (1977) On the diffusion of biological populations. Mathematical Biosciences 33(1-2):35-49

Hadany L, Eshel I, Motro U (2004) No place like home: competition, dispersal and complex adaptation. Journal of Evolutionary Biology 17(6):1328-1336

Hagman M, Phillips BL, Shine R (2008) Tails of enticement: caudal luring by an ambush-foraging snake (Acanthophis praelongus, Elapidae). Functional Ecology:1134-1139

Hambrock R, Lou Y (2009) The evolution of conditional dispersal strategies in spatially heterogeneous habitats. Bulletin of Mathematical Biology 71(8):1793

Hastings A, Petrovskii S, Morozov A (2011) Spatial ecology across scales. Biology Letters 7(2):163

Heil M (2008) Indirect defence via tritrophic interactions. New Phytologist 178(1):41-61

Hillen T, Painter KJ (2009) A user's guide to PDE models for chemotaxis. Journal of Mathematical Biology 58(1-2):183

Holden C (2006) Inching Toward Movement Ecology. Science 313(5788):779-782

Horstmann D (2007) Remarks on some Lotka-Volterra type cross-diffusion models. Nonlinear Analysis: Real World Applications 8(1):90-117

Jansen JE, Van Gorder RA (2018) Dynamics from a predator-prey-quarry-resource-scavenger model. Theoretical Ecology 11(1):19-38

Kareiva P, Odell G (1987). Swarms of predators exhibit "preytaxis" if individual predators use arearestricted search. The American Naturalist 130(2):233-270. 
Kishimoto K (1982) The diffusive Lotka-Volterra system with three species can have a stable non-constant equilibrium solution. Journal of Mathematical Biology 16(1):103-112

Kishimoto K, Weinberger HF (1985) The spatial homogeneity of stable equilibria of some reactiondiffusion systems on convex domains. Journal of Differential Equations 58(1):15 - 21

Kishimoto K, Mimura M, Yoshida K (1983) Stable spatio-temporal oscillations of diffusive Lotka-Volterra system with three or more species. Journal of Mathematical Biology 18(3):213-221

Kondo S, Miura T (2010) Reaction-diffusion model as a framework for understanding biological pattern formation. Science 329(5999):1616-1620

Kondratyev S, Monsaingeon L, Vorotnikov D (2016) A fitness-driven cross-diffusion system from population dynamics as a gradient flow. Journal of Differential Equations 261(5):2784-2808

Krause AL, Burton AM, Fadai NT, Van Gorder RA (2018a) Emergent structures in reaction-advectiondiffusion systems on a sphere. Physical Review E 97(4):042215

Krause AL, Klika V, Woolley TE, Gaffney EA (2018b) Heterogeneity induces spatiotemporal oscillations in reaction-diffusion systems. Physical Review E 97(5):052206

Krause AL, Klika V, Woolley TE, Gaffney EA (2020) From one pattern into another: Analysis of Turing patterns in heterogeneous domains via WKBJ. Journal of the Royal Society Interface, In Press

Krause AL, Van Gorder RA (2020) A non-local cross-diffusion model of population dynamics II: Exact, approximate, and numerical traveling waves.

Kurowski L, Krause AL, Mizuguchi H, Grindrod P, Van Gorder RA (2017) Two-species migration and clustering in two-dimensional domains. Bulletin of Mathematical Biology 79(10):2302-2333

Kuznetsov YA, Antonovsky MY, Biktashev V, Aponina E (1994) A cross-diffusion model of forest boundary dynamics. Journal of Mathematical Biology 32(3):219-232

Laundré JW, Lucina H, William JR (2010) The landscape of fear: ecological implications of being afraid. Open Ecology Journal 3:1-7

Lee JM, Hillen T, Lewis MA (2009). Pattern formation in prey-taxis systems. Journal of biological dynamics 3(6):551-573

Lewis MA, Murray JD (1993) Modelling territoriality and wolf-deer interactions. Nature 366(6457):738

Lewis MA, Maini PK, Petrovskii SV (2013). Dispersal, individual movement and spatial ecology. Lecture Notes in Mathematics (Mathematics Bioscience Series) 2071

Lorenzetti F, Arnason J, Philogene B, Hamilton R (1997) Evidence for spatial niche partitioning in predaceous aphidophaga: use of plant colour as a cue. Entomophaga 42(1-2):49

Lou Y, Ni WM (1996a) Diffusion, self-diffusion and cross-diffusion. Journal of Differential Equations 131(1):79-131

Lou Y, Ni WM (1996b) Diffusion, self-diffusion and cross-diffusion. Journal of Differential Equations 131(1):79-131

Lou Y, Ni WM, Yotsutani S (2015) Pattern formation in a cross-diffusion system. Discrete and Continuous Dynamical Systems 35(4):1589-1607

Lutscher F (2019) Integrodifference Equations in Spatial Ecology, Springer Nature, Cham Switzerland

Lv Y, Yuan R, Pei Y (2013) Turing pattern formation in a three species model with generalist predator and cross-diffusion. Nonlinear Analysis: Theory, Methods \& Applications 85:214-232

Lyson TR, Longrich NR (2010) Spatial niche partitioning in dinosaurs from the latest cretaceous (maastrichtian) of North America. Proceedings of the Royal Society B: Biological Sciences 278(1709):11581164

Ma ZP, Li WT, Wang YX (2017) Spatiotemporal patterns induced by cross-diffusion in a three-species food chain model. International Journal of Bifurcation and Chaos 27(01):1750011

Madin EM, Madin JS, Booth DJ (2011) Landscape of fear visible from space. Scientific Reports 1:14

Malchow H, Petrovskii SV, Venturino E, (2007) Spatiotemporal patterns in ecology and epidemiology: theory, models, and simulation. Chapman and Hall/CRC, London

Matano H, Mimura M (1983) Pattern formation in competition-diffusion systems in nonconvex domains. Publications of the Research Institute for Mathematical Sciences 19(3):1049-1079

Míguez DG, Satnoianu RA, Muñuzuri AP (2006) Experimental steady pattern formation in reactiondiffusion-advection systems. Physical Review E 73(2):025201

Miller PD (1997) Nonmonotone waves in a three species reaction-diffusion model. Methods and Applications of Analysis 4(3):261-282

Mitchell MS and Powell RA (2004) A mechanistic home range model for optimal use of spatially distributed resources. Ecological Modelling 177(1-2):209-232.

Moorcroft PR, Lewis MA. and Crabtree RL (199) Home range analysis using a mechanistic home range model. Ecology 80(5):1656-1665 
Morozov A, Petrovskii S, Li BL (2006) Spatiotemporal complexity of patchy invasion in a predator-prey system with the Allee effect. Journal of Theoretical Biology 238(1):18-35

Mukherjee N, Ghorai S, Banerjee M (2019). Cross-diffusion induced Turing and non-Turing patterns in Rosenzweig-MacArthur model. Letters in Biomathematics, DOI: 10.1080/23737867.2019.1585981

Murray JD (2003) Mathematical Biology II: Spatial Models and Biomedical Applications. Springer, New York

Murrell DJ, Law R (2003) Heteromyopia and the spatial coexistence of similar competitors. Ecology letters 6(1):48-59.

Nakagaki T, Yamada H, Ito M (1999) Reaction-diffusion-advection model for pattern formation of rhythmic contraction in a giant amoeboid cell of the Physarum Plasmodium. Journal of Theoretical Biology 197(4):497-506

Nasreddine E (2012) Well-posedness for a model of individual clustering. Discrete \& Continuous Dynamical Systems-Series B 18(10):2647-2668

Nasreddine E (2014) Two-dimensional individual clustering model. Discrete \& Continuous Dynamical Systems S 7(2):307-316

Nathan R, Muller-Landau HC (2000). Spatial patterns of seed dispersal, their determinants and consequences for recruitment. Trends in ecology \& evolution 15(7):278-285

Nathan R (2008) An emerging movement ecology paradigm. Proceedings of the National Academy of Sciences 105(49):19050-19051

Novak M, Yeakel JD, Noble AE, Doak DF, Emmerson M, Estes JA, Jacob U, Tinker M, Wootton JT (2016) Characterizing species interactions to understand press perturbations: what is the community matrix?. Annual Review of Ecology, Evolution, and Systematics 47:409-432

Nugent C, Quarles W, Solomon T (2004) Experimental studies of pattern formation in a reactionadvection-diffusion system. Physical review letters 93(21):218301

Orr HA (2009) Fitness and its role in evolutionary genetics. Nature Reviews Genetics 10(8):531

Padrón V (1998) Sobolev regularization of a nonlinear ill-posed parabolic problem as a model for aggregating populations. Communications in partial differential equations 23(3-4):457-486

Padrón V (2004) Effect of aggregation on population recovery modeled by a forward-backward pseudoparabolic equation. Transactions of the American Mathematical Society 2739-2756

Page K, Maini PK, Monk NA (2003) Pattern formation in spatially heterogeneous Turing reactiondiffusion models. Physica D: Nonlinear Phenomena 181(1-2):80-101

Page KM, Maini PK, Monk NA (2005) Complex pattern formation in reaction-diffusion systems with spatially varying parameters. Physica D: Nonlinear Phenomena 202(1-2):95-115

Peng R, Wang M, Yang G (2008) Stationary patterns of the Holling-Tanner prey-predator model with diffusion and cross-diffusion. Applied Mathematics and Computation 196(2):570-577

Petrovskii SV, Morozov AY, Venturino E (2002). Allee effect makes possible patchy invasion in a predator-prey system. Ecology Letters 5(3):345-352

Potts JR, Lewis MA (2014) How do animal territories form and change? Lessons from 20 years of mechanistic modelling. Proceedings of the Royal Society B: Biological Sciences 281(1784):20140231

Potts JR, Lewis MA (2019) Spatial memory and taxis-driven pattern formation in model ecosystems. Bulletin of mathematical biology 81:2725-2747

Quillfeldt P, Masello JF, Navarro J, Phillips RA (2013) Year-round distribution suggests spatial segregation of two small petrel species in the South Atlantic. Journal of Biogeography 40(3):430-441

Riaz SS, Kar S, Ray DS (2005) Differential flow induced transition of Hopf instability to Turing instability and pattern formation. Physica D: Nonlinear Phenomena 203(3-4):224-232

Richardson JL, Urban MC, Bolnick DI, Skelly DK (2014) Microgeographic adaptation and the spatial scale of evolution. Trends in Ecology \& Evolution 29(3):165-176

Rovinsky AB, Menzinger M (1992) Chemical instability induced by a differential flow. Physical Review Letters 69(8):1193

Rowell JT (2009) The limitation of species range: a consequence of searching along resource gradients. Theoretical Population Biology 75(2-3):216-227

Ruxton GD, Rohani P (1999) Fitness-dependent dispersal in metapopulations and its consequences for persistence and synchrony. Journal of Animal Ecology 68(3):530-539

Satnoianu RA (2003) Coexistence of stationary and traveling waves in reaction-diffusion-advection systems. Physical Review E 68(3):032101

Satnoianu RA, Menzinger M, Maini PK (2000) Turing instabilities in general systems. Journal of Mathematical Biology 41(6):493-512 
Schuette P, Wagner AP, Wagner ME, Creel S (2013) Occupancy patterns and niche partitioning within a diverse carnivore community exposed to anthropogenic pressures. Biological Conservation 158:301-312

Shepard EL, Wilson RP, Rees WG, Grundy E. Lambertucci SA, Vosper SB (201) Energy landscapes shape animal movement ecology. The American Naturalist, 182(3):298-312

Sherratt JA (2011) Pattern solutions of the Klausmeier model for banded vegetation in semi-arid environments II: patterns with the largest possible propagation speeds. Proceedings of the Royal Society A: Mathematical, Physical and Engineering Sciences 467(2135):3272-3294

Sherratt JA, Eagan BT, Lewis MA (1997) Oscillations and chaos behind predator-prey invasion: mathematical artifact or ecological reality? Philosophical transactions of the Royal Society of London Series B: Biological Sciences 352(1349):21-38

Shigesada N, Kawasaki K, Teramoto E (1979) Spatial segregation of interacting species. Journal of Theoretical Biology 79(1):83-99

Strobl MAR, Krause AL, Damaghi M, Gillies R, Anderson ARA, Maini PK (2019) Mix \& Match: Phenotypic coexistence as a key facilitator of solid tumour invasion. Bulletin of Mathematical Biology, In Press

Taylor P, Crewe T Mackenzie S, Lepage D, Aubry Y, Crysler Z, Finney, G, Francis C, Guglielmo C, Hamilton D, and Holberton R, 2017. The Motus Wildlife Tracking System: a collaborative research network to enhance the understanding of wildlife movement. Avian Conservation and Ecology 12(1):8

Tian C, Ling Z, Lin Z (2011) Turing pattern formation in a predator-prey-mutualist system. Nonlinear Analysis: Real World Applications 12(6):3224-3237.

Turing AM (1952) The chemical basis of morphogenesis. Philosophical Transactions of the Royal Society of London Series B, Biological Sciences 237(641):37-72

Wang W, Lin Y, Zhang L, Rao F, Tan Y (2011) Complex patterns in a predator-prey model with self and cross-diffusion. Communications in Nonlinear Science and Numerical Simulation 16(4):2006-2015

Wen Z, Fu S (2016) Turing instability for a competitor-competitor-mutualist model with nonlinear crossdiffusion effects. Chaos, Solitons \& Fractals 91:379-385

Winder M (2009) Photosynthetic picoplankton dynamics in Lake Tahoe: temporal and spatial niche partitioning among prokaryotic and eukaryotic cells. Journal of Plankton Research 31(11):1307-1320

Zhao Q, Van den Brink PJ, Carpentier C, Wang YX, Rodríguez-Sánchez P, Xu C, Vollbrecht S, Gillissen F, Vollebregt M, Wang S, De Laender F (201) Horizontal and vertical diversity jointly shape food web stability against small and large perturbations. Ecology letters 22(7):1152-1162 